\title{
Action of the conformal group on steady state solutions to Maxwell's equations and background radiation
}

\author{
Bertram Kostant and Nolan Wallach
}

\begin{abstract}
The representation of the conformal group $(P S U(2,2))$ on the space of solutions to Maxwell's equations on the conformal compactification of Minkowski space is shown to break up into four irreducible unitarizable smooth Fréchet representations of moderate growth. An explicit inner product is defined on each representation. The frequency spectrum of each of these representations is analyzed. These representations have notable properties; in particular they have positive or negative energy, they are of type $A_{\mathfrak{q}}(\lambda)$ and are quaternionic. Physical implications of the results are explained.
\end{abstract}

\section{Introduction}

The purpose of this paper is to analyze the steady state solutions of Maxwell's equations in a vacuum using the tools of representation theory. By steady state we mean those solutions that extend to the conformal compactification of Minkowski space. That is, we look upon the solutions of Maxwell's equations as tensor valued on

$\mathbb{R}^{4}$ with the flat Lorentzian metric given by

$$
-d x_{1}^{2}-d x_{2}^{2}-d x_{3}^{2}+d t^{2}
$$

Key words and phrases: Maxwell's equations, conformal compactification, conformal group, unitary representation

MSC 2010 : 78A25, 58Z05, 22E70, 22E45.

Research partially supported by NSF grant DMS 0963035. 
where $x_{1}, x_{2}, x_{3}$ and $x_{4}=t$ yield the standard coordinates of $\mathbb{R}^{4} . \mathbb{R}^{4}$ with this metric will be denoted by $\mathbb{R}^{1,3}$. The conformal compactification is the space $S^{3} \times\{ \pm 1\} S^{1}$ (modulo the product action of \pm 1 ) with (up to a positive scalar multiple on both factors) the product metric with the negative of the constant curvature 1 metric on $S^{3}$ and the usual metric on $S^{1}$. The injection, $f$, of $\mathbb{R}^{4}$ into $S^{3} \times_{\{ \pm 1\}} S^{1}$ is the inverse of a variant of stereographic projection (see Section 2). This embedding is not an isometry, but it is conformal. Our approach to Maxwell's equations uses the equivalent formulation in terms of differential 2-forms on Minkowski space. More generally, if $(M, g)$ is an oriented Lorentzian four manifold (signature $(-,-,-,+)$ ) and if "* $*$ denotes the Hodge star operator on 2 -forms relative to the volume form, $\gamma$, with $g(\gamma, \gamma)=-1$, then there is a version of Maxwell's equations on $\Omega^{2}(M)$ (differential two forms) given by

$$
d \omega=d * \omega=0 .
$$

Let $M$ and $N$ be four-dimensional Lorentzian manifolds, let $F: M \rightarrow N$ be a conformal transformation, and let $\omega$ be a solution to Maxwell's equations on $N$. Then $F^{*} \omega$ is a solution to Maxwell's equations on $M$. Since $f$ is conformal we see that the pullback of solutions to Maxwell's equations on $S^{3} \times_{\{ \pm 1\}} S^{1}$ yields solutions to the usual Maxwell equations.

The group of conformal transformations of $S^{3} \times_{\{ \pm 1\}} S^{1}$ is locally the group $S O(4,2)$ and thus the solutions to Maxwell's equations on $S^{3} \times_{\{ \pm 1\}} S^{1}$ form a representation of this group. We can interpret this as follows: We first note that we can replace $S^{3} \times_{\{ \pm 1\}} S^{1}$ with the group $U(2)$. If on $\operatorname{Lie}(U(2))$ we put the Lorentzian form that corresponds to the quadratic form $-\operatorname{det} X$, then the corresponding bi-invariant metric on a $U(2)$ is isometric, up to positive scalar multiple, with $S^{3} \times_{\{ \pm 1\}} S^{1}$. We interpret this space as the Shilov boundary of the Hermitian symmetric space that corresponds to $G=S U(2,2)$ (which is locally isomorphic with $S O(4,2))$.

We denote by Maxw the space of solutions in $\Omega^{2}(U(2))$ to Maxwell's equations. We show that there is a canonical nondegenerate $G$-invariant Hermitian form on Maxw. Further, we show that as a smooth Fréchet representation of $G$, Maxw splits into the direct sum of four irreducible (Fréchet) representations (of moderate growth) that are mutually orthogonal relative to the form. This form is positive definite on two of the irreducible pieces and negative definite on the other two. Since the $K=S(U(2) \times U(2))$ isotypic components of Maxw are all finite dimensional we see that this yields 
four unitary irreducible representations of $G$. Two of the representations are holomorphic (negative energy in the physics literature) and two are antiholomorphic (positive energy). We also describe them in terms of the $A_{\mathfrak{q}}(\lambda)$ that yield second continuous cohomology (the four theta stable parabolics $\mathfrak{q}$ involved relate these representations to twister theory) and in terms of quaternionic representations $(S U(2,2)$ is the quaternionic real form of $S L(4, \mathbb{C}))$. These representations are actually representations of $P S U(2,2)$. In this group there is a dual pair $P S U(1,1), S O(3)$ establishing an analogue of Howe duality in each of the four representations. The realization of these representations is intimately related to the work in $[\mathrm{K}]$.We use the decomposition of the restriction of these representations to $P S U(1,1)$ to analyze the frequency distribution of the solutions in each of the $P S U(2,2)$ representations.

We interpret the plane wave solutions as generalized Whittaker vectors on Maxw and the solutions as wave packets of the Whittaker vectors. These wave packets have constrained frequency spectrum and using Planck's black body radiation law the frequency limitation and luminosity of the corresponding radiation determines the temperature to narrow constraints. This means that we can fit our solutions to the measured background radiation on a steady state universe. We make no assertions as to how such a steady state universe might physically exist. There are many suggestions in the literature (e.g., the work of Hoyle et al $[\mathrm{HBN}]$ ). All seem complicated. However, we will content ourselves to the assertion that the big bang is not necessarily the only possible interpretation of background radiation.

There is also an interpretation of the red shift that can be gleaned from this work involving the relationship between the measurement of time from the proposed "big bang" and the steady state "time" which is periodic but with a large period appearing to move faster as we look backwards or forwards in terms of "standard" time. (See [S].)

We are aware that many of the aspects of representation theory in this paper could have been done in more generality. We have constrained our attention to the four representations at hand since the main thrust of this paper is to show how representation theory can be used to study well-known equations in physics.

Parts of this work should be considered expository. Related work has been done by [HSS] on the action of the conformal group on solutions of the wave equation and $[\mathrm{EW}]$ relating positive energy representations to generalized Dirac equations. 


\section{Conformal compactification of Minkowski space}

Let $\mathbb{R}^{1,3}$ denote $\mathbb{R}^{4}$ with the pseudo-Riemannian (Lorentzian) structure given by $(x, y)=-x_{1} y_{1}-x_{2} y_{2}-x_{3} y_{3}+x_{4} y_{4}$. Here $x_{i}, i=1,2,3,4$, are the standard coordinates on $\mathbb{R}^{4}$ and we identify the tangent space at every point with $\mathbb{R}^{4}$. We can realize this space as the space of $2 \times 2$ Hermitian matrices (a 4-dimensional vector space over $\mathbb{R}$ ), $V$, with the Lorentzian structure corresponding to the quadratic form given by the determinant. Note that

$$
\operatorname{det}\left[\begin{array}{cc}
x_{4}+x_{3} & x_{1}+i x_{2} \\
x_{1}-i x_{2} & x_{4}-x_{3}
\end{array}\right]=(x, x) .
$$

We can also realize the space in terms of skew Hermitian matrices $\mathfrak{u}(2)=$ $i V$ and noting that the form becomes - det. With this interpretation, and realizing that $\mathfrak{u}(2)=\operatorname{Lie}(U(2))$ we have an induced Lorentzian structure, $\langle\ldots, \ldots\rangle$ on $U(2)$. We also have a transitive action of $K=U(2) \times U(2)$ on $U(2)$ by right and left translation

$$
\left(g_{1}, g_{2}\right) u=g_{1} u g_{2}^{-1}
$$

Since the isotropy group at $I$ is $M=\operatorname{diag}(\mathrm{U}(2))=\{(\mathrm{g}, \mathrm{g}) \mid \mathrm{g} \in \mathrm{U}(2)\}$. We see that $K$ acts by isometries on $U(2)$ with this structure.

We will now consider a much bigger group that acts. We first consider the indefinite unitary group $G \cong U(2,2)$ given by the elements, $g \in M_{4}(\mathbb{C})$ such that

$$
g\left[\begin{array}{cc}
0 & i I_{2} \\
-i I_{2} & 0
\end{array}\right] g^{*}=\left[\begin{array}{cc}
0 & i I_{2} \\
-i I_{2} & 0
\end{array}\right]
$$

Here as usual $g^{*}$ means conjugate transpose. $G \cap U(4)$ is the group of all matrices of the $2 \times 2$ block form

$$
\left[\begin{array}{cc}
A & B \\
-B & A
\end{array}\right]
$$

satisfying $A B^{*}=B A^{*}$ and $A A^{*}+B B^{*}=I$. These equations are equivalent to the condition

$$
A+i B \in U(2)
$$

It is easy to see that the map

$$
\Psi: G \cap U(4) \rightarrow U(2) \times U(2)
$$


given by

$$
\left[\begin{array}{cc}
A & B \\
-B & A
\end{array}\right] \mapsto(A-i B, A+i B)
$$

defines a Lie group isomorphism. This leads to the action of $G \cap U(4)$ on $U(2)$ given by

$$
\left[\begin{array}{cc}
A & B \\
-B & A
\end{array}\right] \cdot x=(A-i B) x(A+i B)^{-1} .
$$

Note that the stabilizer of $I_{2}$ is the subgroup isomorphic with $M$ given by the elements

$$
\left[\begin{array}{cc}
A & 0 \\
0 & A
\end{array}\right], A \in U(2) .
$$

We extend this to a map of $G$ to $U(2)$ given by

$$
\Phi:\left[\begin{array}{cc}
A & B \\
C & D
\end{array}\right] \mapsto(A+i C)(A-i C)^{-1}
$$

This makes sense since $A-i C$ is invertible if $\left[\begin{array}{cc}A & B \\ C & D\end{array}\right] \in G$. We consider the subgroup $P$ of $G$ that consists of the matrices

$$
\left[\begin{array}{cc}
g & g X \\
0 & \left(g^{*}\right)^{-1}
\end{array}\right]
$$

with $g \in G L\left(2, \mathbb{C}\right.$ ) and $X \in H$ (in other words, $X^{*}=X$ ). Then every element of $G$ can be written in the form $k p$ with $k \in G \cap U(4)$ and $p \in P$. We note that $\Phi(k p)=\Phi(k)=k \cdot I$. Now, $U(4) \cap K$ acts transitively on $G / P$ and the stabilizer of the identity coset is the group $\left[\begin{array}{cc}A & 0 \\ 0 & A\end{array}\right], A \in U(2)$. We will identify $K$ with $U(4) \cap G$ (under $\Psi$ ) and $M$ with the stabilizer of the identity. Thus $G / P=K / M$.

We consider the subgroup $\bar{N}$ :

$$
\left[\begin{array}{ll}
I & 0 \\
Y & I
\end{array}\right], Y^{*}=Y
$$

If we write

$$
\left[\begin{array}{ll}
I & 0 \\
Y & I
\end{array}\right]=k p
$$


with

$$
k=\left[\begin{array}{cc}
A & B \\
-B & A
\end{array}\right]
$$

as above, then $A \in G L(2, \mathbb{C})$ and

$$
-B A^{-1}=Y
$$

One can see that if we set

$$
k(Y)=\left[\begin{array}{ll}
\frac{I}{\sqrt{I+Y^{2}}} & \frac{-Y}{\sqrt{I+Y^{2}}} \\
\frac{Y}{\sqrt{I+Y^{2}}} & \frac{I}{\sqrt{I+Y^{2}}}
\end{array}\right],
$$

then

$$
k(Y) P=\left[\begin{array}{cc}
I & 0 \\
Y & I
\end{array}\right] P .
$$

This gives an embedding of $H$ into $U(2)$

$$
Y \longmapsto(I+i Y)(I-i Y)^{-1},
$$

the Cayley transform. We next explain how this is related to the Cayley transform in the sense of bounded symmetric domains.

We note that it is more usual to look upon $G$ (in its more usual incarnation) as the group of all elements $g \in G L(4, \mathbb{C})$ such that

$$
g\left[\begin{array}{cc}
I & 0 \\
0 & -I
\end{array}\right] g^{*}=\left[\begin{array}{cc}
I & 0 \\
0 & -I
\end{array}\right] .
$$

Let us set $G_{1}$ equal to this group. The relationship between the two groups is given as follows. Set

$$
L=\frac{1}{\sqrt{2}}\left[\begin{array}{cc}
I & i I \\
I & -i I
\end{array}\right]
$$

(a unitary matrix) if

$$
\sigma(g)=L g L^{*}
$$

then $\sigma$ defines an isomorphism of $G$ onto $G_{1}$. $G_{1}$ has an action by linear fractional transformations on the bounded domain, $\mathbf{D}$, given as the set of all $Z \in M_{2}(\mathbb{C})$ such that $Z Z^{*}<I$ (here $<$ is the order defined by the cone of positive definite Hermitian matrices. If $g=\left[\begin{array}{cc}A & B \\ C & D\end{array}\right] \in G_{1}$, then

$$
g \cdot Z=(A Z+B)(C Z+D)^{-1} \text {. }
$$


We note that if $Y^{*}=Y$ then

$$
\sigma\left(\left[\begin{array}{cc}
I & 0 \\
Y & I
\end{array}\right]\right) \cdot I=(I+i Y)(I-i Y)^{-1}
$$

and

$$
\sigma\left(\left[\begin{array}{cc}
I & Y \\
0 & I
\end{array}\right]\right) \cdot I=I
$$

The embedding $F$ of Minkowski space $\mathbb{R}^{1,3}$ into $U(2)$ given by

$$
\left(x_{1}, x_{2}, x_{3}, x_{4}\right) \mapsto\left[\begin{array}{cc}
x_{4}+x_{3} & x_{1}+i x_{2} \\
x_{1}-i x_{2} & x_{4}-x_{3}
\end{array}\right]=X \longmapsto(I+i X)(I-i X)^{-1}
$$

embeds it as a dense open subset. However, it is only a conformal embedding. Indeed

Lemma 1 The embedding $F$ is conformal with

$$
\left(F^{*}\langle\ldots, \ldots\rangle\right)_{x}=4\left(1+2 \sum x_{i}^{2}+(x, x)^{2}\right)^{-1}(\ldots, \ldots)_{x} .
$$

Proof. We note that $T_{u}(U(2))=\{u X \mid X \in \mathfrak{u}(2)\}$. Furthermore, $\langle u X, u X\rangle_{u}=$ $-\operatorname{det}(X)$. Now let $Y \in M_{2}(\mathbb{C})$ be such that $Y^{*}=Y$, that is $Y \in H$. Let $Q(Y)=(I+i Y)(I-i Y)^{-1}$. We calculate $\left\langle d Q_{Y}(v), d Q_{Y}(v)\right\rangle_{Q(Y)}$ for $v \in H$ thought of as being an element of $T_{Y}(H)$. We get

$$
\begin{gathered}
d Q_{Y}(v)=i v(I-i Y)^{-1}+(I+i Y)(I-i Y)^{-1} i v(I-i Y)^{-1} \\
=i(I+i Y)(I-i Y)^{-1}\left((I-i Y)(I+i Y)^{-1}+I\right) v(1-i Y)^{-1} \\
=2 i Q(Y)(I+i Y)^{-1} v(1-i Y)^{-1} .
\end{gathered}
$$

Thus

$$
\left\langle d Q_{Y}(v), d Q_{Y}(v)\right\rangle_{Q(Y)}=4 \frac{\operatorname{det}(v)}{\operatorname{det}\left(I+Y^{2}\right)}=4 \frac{(v, v)_{Y}}{\operatorname{det}\left(I+Y^{2}\right)} .
$$

Now calculate $\operatorname{det}\left(I+Y^{2}\right)$ in terms of the $x_{i}$.

More generally we have

Lemma 2 The action of $G\left(\right.$ or $\left.G_{1}\right)$ on $U(2)$ given by the linear fractional transformations is conformal relative to the pseudo-Riemannian metric $\langle\ldots, \ldots\rangle$ on $U(2)$. 
Proof. Let

$$
\phi(Z)=g \cdot Z=(A Z+B)(C Z+D)^{-1}
$$

with $g \in G_{1}$. Then if $X \in \mathfrak{u}(2)$ we have

$$
\begin{gathered}
d \phi_{Z}(Z X)=(A Z X-\phi(Z) C Z X)(C Z+D)^{-1} \\
=\phi(Z)\left(\phi(Z)^{-1} A Z X-C Z X\right)(C Z+D)^{-1} .
\end{gathered}
$$

Thus since $-\operatorname{det} X=\langle Z X, Z X\rangle_{Z}$ we have $\left\langle d \phi_{Z}(Z X), d \phi_{Z}(Z X)\right\rangle_{\phi(Z)}=$

$$
\operatorname{det}\left(\left(\phi(Z)^{-1} A Z-C Z\right)(C Z+D)^{-1}\right)\langle Z X, Z X\rangle_{Z}
$$

This proves the conformality.

We note that

$$
\begin{gathered}
\left(\phi(Z)^{-1} A Z X-C Z X\right)(C Z+D)^{-1} \\
=(C Z+D)\left((A Z+B)^{-1} A Z-(C Z+D)^{-1} C Z\right)(C Z+D)^{-1} .
\end{gathered}
$$

Thus the conformal factor is

$$
\begin{aligned}
& \operatorname{det}\left((A Z+B)^{-1} A Z-(C Z+D)^{-1} C Z\right) \\
& =\operatorname{det}\left((A Z+B)^{-1} B-(C Z+D)^{-1} D\right) .
\end{aligned}
$$

\section{Maxwell's equations on compactified Minkowski space}

We will first recall Maxwell's equations in Lorentzian form. For this we need some notation. If $M$ is a smooth manifold, then $\Omega^{k}(M)$ will denote the space of smooth $k$-forms on $M$. We note that if $(M, g)$ is an $n$-dimensional pseudo-Riemannian manifold then $g$ induces nondegenerate forms on each fiber $\wedge^{k} T(M)_{x}^{*}$ which we will also denote as $g_{x}$. If $M$ is oriented then there is a unique element $\gamma \in \Omega^{n}(M)$ such that if $x \in M$ and $v_{1}, \ldots, v_{n}$ is an oriented pseudo-orthonormal basis of $T(M)_{x}$ (i.e., $\left|g_{x}\left(v_{i}, v_{j}\right)\right|=\delta_{i j}$ ) then $\gamma_{x}\left(v_{1}, \ldots, v_{n}\right)=1$. Using $\gamma$ we can define the Hodge $*$ operator on $M$ as follows: If $\omega \in \wedge^{k} T(M)_{x}^{*}$, then $* \omega$ is defined to be the unique element of $\wedge^{n-k} T(M)_{x}^{*}$ such that $\eta \wedge * \omega=g_{x}(\eta, \omega) \gamma_{x}$ for all $\eta \in \wedge^{k} T(M)_{x}^{*}$.

The next result is standard. 
Lemma 3 Let $F: M \rightarrow N$ be a conformal, orientation, preserving mapping of oriented pseudo-Riemannian manifolds. If $\operatorname{dim} M=\operatorname{dim} N=2 k$ then $F^{*} * \omega=* F^{*} \omega$ for $\omega \in \Omega^{k}$.

With this notation in place we can set up Maxwell's equations. Take $t=x_{4}$ in $\mathbb{R}^{1,3}$ and let $\omega \longmapsto * \omega$ denote the Hodge star operator on differential forms with respect to the Lorentzian structure $(\ldots, \ldots)$ and the orientation corresponding to $\gamma=d x_{1} \wedge d x_{2} \wedge d x_{3} \wedge d t$. Then Maxwell's equations in an area free of current (simple media e.g., in a vacuum, with the dielectric constant, the permeability and thus the speed of light normalized to 1) can be expressed in terms of 2 -forms as

$$
d \omega=d * \omega=0
$$

with $d$ the exterior derivative. We note that in this formulation if $\mathbf{E}=$ $\left(e_{1}, e_{2}, e_{3}\right)$ and $\mathbf{H}=\left(h_{1}, h_{2} . h_{3}\right)$ are respectively the electric field intensity and the magnetic field intensity vectors, then

$$
\begin{gathered}
\omega=h_{1} d x_{2} \wedge d x_{3}-h_{2} d x_{1} \wedge d x_{3}+h_{3} d x_{1} \wedge d x_{2} \\
-e_{1} d x_{1} \wedge d t-e_{2} d x_{2} \wedge d t-e_{3} d x_{3} \wedge d t .
\end{gathered}
$$

The equations (1) are then the same as

$$
\nabla \cdot \mathbf{E}=\nabla \cdot \mathbf{H}=0
$$

and

$$
\frac{\partial}{\partial t} \mathbf{E}=-\nabla \times \mathbf{H}, \frac{\partial}{\partial t} \mathbf{H}=\nabla \times \mathbf{E} .
$$

Here the $*$ operation is just $\mathbf{E} \rightarrow \mathbf{H}$ and $\mathbf{H} \rightarrow-\mathbf{E}$, which is the duality between electricity and magnetism in the physics literature.

We note that if $M=\mathbb{R}^{1,3}$ and $N=U(2)$ with the Lorentzian structures described in the previous section, if $F$ is the map described above and if $\omega \in \Omega^{2}(U(2))$ satisfies the equations (1), then $F^{*} \omega$ satisfies Maxwell's equations on $\mathbb{R}^{1,3}$. We will thus call the equations (1) Maxwell's equations on compactified Minkowski space.

The group $G$ (or $G_{1}$ ) acts on $U(2)$ by conformal diffeomorphisms. Thus we see that the space of solutions to Maxwell's equations defines a representation of $G$.(which acts by pullback). Most of the rest of this article will be devoted to that analysis of this representation. 
Denote by $\Omega^{k}(U(2))_{\mathbb{C}}$ the complex valued $k$-forms. Endow it with the $C^{\infty}$ topology which is a Fréchet space structure and the corresponding action of $G$ on $\Omega^{2}(U(2))$ defines it as a smooth Fréchet representation of $G$ moderate growth. To see this, we note that as a $G$-homogeneous space $U(2) \cong G / P$. Let $\mu$ denote the isotropy action of $P$ on $V=T_{I P}(G / P)_{\mathbb{C}}$ (i.e., the action of $P$ on $\operatorname{Lie}(G) / \operatorname{Lie}(P) \otimes \mathbb{C})$. Then the space $\Omega^{k}(U(2))_{\mathbb{C}}$ with the $C^{\infty}$ topology and $G$ action by pullback is just the $C^{\infty}$ induced representation

$$
\operatorname{Ind}_{\mathrm{P}}^{\mathrm{G}}\left(\wedge^{\mathrm{k}} \mathrm{V}^{*}\right)^{\infty} .
$$

Furthermore, since it is as a $K$-representation

$$
\operatorname{Ind}_{\mathrm{M}}^{\mathrm{K}}\left(\wedge^{\mathrm{k}} \mathrm{V}^{*}\right)^{\infty},
$$

Frobenius reciprocity implies that the representation is admissible (that is, the multiplicities of the $K$-types is finite). The maps $d$ and $d *$ are continuous maps in this topology to $\Omega^{3}(U(2))_{\mathbb{C}}$; thus the solutions of Maxwell's equations on $U(2)$ define an admissible, smooth Fréchet representation of moderate growth.

\section{The $K$-isotypic components of the space of solutions to Maxwell's equations on compactified Minkowski space: step 1}

In this section we will begin determination of the $K$-isotypic components of the space of solutions to Maxwell's equations. We will proceed by first determining the isotypic components of ker $d$ on $\Omega^{2}(U(2))_{\mathbb{C}}$. We will then use explicit calculations for the case at hand of $d$ and the Hodge star operator to complete the picture. We will now begin the first step.

We note that $U(2)$ is diffeomorphic with $S U(3) \times S^{1}$ under the map

$$
u, z \mapsto u\left[\begin{array}{cc}
z & 0 \\
0 & 1
\end{array}\right]
$$

with $u \in S U(2)$ and $z \in S^{1}=\{z \in \mathbb{C}|| z \mid=1\}$. We note that $S U(2)$ is diffeomorphic with $S^{3}$ which implies that we have

$$
H^{1}(U(2), \mathbb{C})=\mathbb{C},
$$




$$
H^{2}(U(2), \mathbb{C})=0
$$

and

$$
H^{3}(U(2), \mathbb{C})=\mathbb{C} .
$$

So de Rham's theorem implies that we have the following short exact sequences

$$
\begin{aligned}
& 0 \rightarrow \mathbb{C} 1 \rightarrow C^{\infty}(U(2), \mathbb{C}) \rightarrow \operatorname{ker} d_{\mid \Omega^{1}(U(2))_{\mathbb{C}}} \rightarrow \mathbb{C} \mu \rightarrow 0 ; \\
& 0 \rightarrow \operatorname{ker} d_{\mid \Omega^{1}(U(2))} \rightarrow \Omega^{1}(U(2))_{\mathbb{C}} \rightarrow \operatorname{ker} d_{\mid \Omega^{2}(U(2))_{\mathbb{C}}} \rightarrow 0
\end{aligned}
$$

in both sequences the map to the kernel is given by $d$. Also $\mu$ is the image of det* $\frac{d z}{z}$ in the quotient space. These are all morphisms of smooth Fréchet representations of $G$ of moderate growth.

We also note that the center of $G$ consists of the multiples of the identity and so acts trivially on $U(2)$. Now $K=U(2) \times U(2)$ and the multiples of the identity correspond under this identification with the diagonal elements $C=\{(z I, z I)|| z \mid=1\}$ and $M$ is the diagonal $U(2)$ in $K$. The actual groups acting on $U(2)$ are $K / C$ and $M / C$. We define $K_{1}=S U(2) \times U(2)$ and $M_{1}=\{(u, u) \mid u \in S U(2)\}$. Then under the natural map $K / M=K_{1} / M_{1}$ we still have a redundancy of $\mu_{2}=\{ \pm(I, I)\}$. We will use the notation $\left(\tau_{p, q, r}, F^{(p, q, r)}\right)$ for the representation of $K_{1}$ on $S^{p}\left(\mathbb{C}^{2}\right) \otimes S^{q}\left(\mathbb{C}^{2}\right)\left(S^{p}\left(\mathbb{C}^{2}\right)\right.$ the $p^{t h}$ symmetric power) given by $\tau_{p, q, r}(u, v z)=z^{r} S^{p}(u) \otimes S^{q}(u)$ where $p, q \in \mathbb{Z}_{\geq 0}$, $r \in \mathbb{Z}$ and $r \equiv q \bmod 2$. If $V$ is a closed $K$-invariant subspace of $\Omega^{k}(U(2))_{\mathbb{C}}$ then we denote by $V_{p, q, r}$ its $\tau_{p, q, r}$ isotypic component.

Lemma 4 As a representation of $K_{1}$, the space of $K_{1}$-finite vectors of ker $d_{\mid \Omega^{2}(U(2))_{\mathbb{C}}}$ splits into a direct sum

$$
\begin{aligned}
& \bigoplus_{\substack{k \geq 0 \\
r \equiv k \bmod 2}}\left(F^{k+2, k, r} \oplus F^{k, k+2, r} \oplus F^{k+1, k+1, r}\right) . \\
&
\end{aligned}
$$

Furthermore, if $p-q \neq 0$ then

$$
d: \Omega^{1}(U(2))_{p, q, r} \rightarrow\left(\operatorname{ker} d_{\mid \Omega^{2}(U(2))_{\mathbb{C}}}\right)_{p, q, r}
$$

is a bijective $K$-intertwining operator. 
Proof. The Peter-Weyl theorem implies that $L^{2}(U(2))$ is a Hilbert space direct sum

$$
\bigoplus_{\tau \in \widehat{U(2)}} V^{\tau} \otimes\left(V^{\tau}\right)^{*}
$$

where $\widehat{U(2)}$ is the set of equivalence classes of irreducible finite-dimensional representations of $U(2)$ and $V^{\tau}$ is a choice of representative of $\tau$. We have the exact sequence

$$
1 \rightarrow\{ \pm(I, 1)\} \rightarrow S U(2) \times S^{1} \rightarrow U(2) \rightarrow 1
$$

with the last map $u, z \longmapsto z u$. This implies (as above) that if we define $V^{p, r}$ to be the representation, $\tau_{p, r}$ of $S U(2) \times S^{1}$ on $S^{p}\left(\mathbb{C}^{2}\right)$ with $\tau_{p, r}(u, z) v=z^{r} S^{p}(u) v$, then $\tau_{p, r}$ is the lift of an irreducible representation of $U(2)$ if and only if $r \equiv p \bmod 2$. These representations give a complete set of representatives for $\widehat{U(2)}$. We note that the dual representation of $\tau_{p, r}$ is equivalent with $\tau_{p,-r}$. We therefore see that the space of $K$-finite vectors in $C^{\infty}(U(2))_{\mathbb{C}}$ is isomorphic with the direct sum

$$
\bigoplus_{\substack{p \in \mathbb{Z}_{\geq 0} \\ r \equiv p \bmod 2}} F^{p, p, r} .
$$

We now apply Frobenius reciprocity to analyze the isotypic components of $\Omega^{1}(U(2))_{\mathbb{C}}$. As we have noted as a representation of $K$ it is just the smooth induced representation of $M$ to $K$ where

$$
M=\Delta(U(2))=\{(u, u) \mid u \in U(2)\}
$$

is acting on $\operatorname{Lie}(U(2))_{\mathbb{C}}$ under $\operatorname{Ad}(u)$. Thus in terms of the parameters above (identifying $M$ with $U(2))$ we have

$$
\operatorname{Lie}(U(2))_{\mathbb{C}} \cong F^{0,0} \oplus F^{2,0} .
$$

Now Frobenius reciprocity implies that

$$
\begin{gathered}
\operatorname{dim} \operatorname{Hom}_{K}\left(F^{p, q, r}, \Omega^{1}(U(2))_{\mathbb{C}}\right)=\operatorname{dim} \operatorname{Hom}_{M}\left(F^{p, q, r}, F^{0,0}\right) \\
+\operatorname{dim} \operatorname{Hom}_{M}\left(F^{p, q, r}, F^{2,0}\right)
\end{gathered}
$$


The argument above says that $\operatorname{dim} \operatorname{Hom}_{M}\left(F^{p, q, r}, F^{0,0}\right)=0$ unless $p=q$ and $r \equiv p \bmod 2$. Now the Clebsch-Gordan formula implies that

$$
F_{\mid M}^{p, q, r} \cong \bigoplus_{j=0}^{\min (p, q)} F^{p+q-2 j, r}
$$

This implies that $\operatorname{dim} \operatorname{Hom}_{M}\left(F^{p, q, r}, F^{2,0}\right)=0$ unless $p=q$ or $|p-q|=2$ and in either of these cases it is 1 .Now the exact sequences (1) and (2) above imply the theorem.

Remark 5 In the physics literature if $\omega$ is a solution to Maxwell's equations (as in the beginning of Section 3), then a one-form $\beta$ such that $d \beta=\omega$ yields in the $\mathbf{E}, \mathbf{H}$ formulation a potential $\mathbf{A}$. In our formulation if we pull back to Minkowski space and we write

$$
\beta=\sum_{i=1}^{4} a_{i} d x_{i}
$$

then considering $t=x_{4}$ and writing $\phi=a_{4}$ and $\mathbf{A}=\left(a_{1}, a_{2}, a_{3}\right)$ we then have

$$
\mathbf{E}=\nabla \times \mathbf{A}, \mathbf{H}=-\frac{\partial \mathbf{A}}{\partial t}+\nabla \phi .
$$

This is the dual of what one normally finds in the physics literature. It is pointed out that this potential has the ambiguity of a gradient field. We will see that the only isotypic components of Maxwell's equations are $\tau_{p, q, r}$ with $|p-q|=2$ and $r= \pm(\max (p, q))$. Thus using only those Peter-Weyl coefficients yields a unique potential.

We will use the above lemma and some direct calculations to describe the $K$-isotypic components of Maxwell's equations in the next section.

\section{The $K$-isotypic components of the space of solutions to Maxwell's equations on compactified Minkowski space: step 2}

We retain the notation of the previous section. Let $x_{4}=i I$ and

$$
x_{1}=\left[\begin{array}{cc}
i & 0 \\
0 & -i
\end{array}\right], x_{2}=\left[\begin{array}{cc}
0 & 1 \\
-1 & 0
\end{array}\right], x_{3}=\left[\begin{array}{cc}
0 & i \\
i & 0
\end{array}\right] \text {. }
$$


We will use the usual identification of $\operatorname{Lie}(U(2))$ (left invariant vector fields) with skew-Hermitian $2 \times 2$ matrices (which we denote, as is usual, by $\mathfrak{u}(2)$ ). Thus if $x \in \mathfrak{u}(2)$ then $x_{u}$ is the tangent vector at 0 to the curve $t \longmapsto u e^{t x}$. We note that $\left(\left(x_{j}\right)_{u},\left(x_{k}\right)_{u}\right)_{u}=\varepsilon_{j} \delta_{j, k}$ with $\varepsilon_{j}=-(-1)^{\delta_{j 4}}$. Thus $x_{1}, x_{2}, x_{3}, x_{4}$ define a pseudo-orthonormal frame on $U(2)$. We use this frame to define $\gamma$. Since there will be many uses of the star operator and pullbacks we will use the notation $J \omega=* \omega$ for $\omega \in \wedge^{2} T^{*}(U(2))_{u}$ for all $u \in U(2)$. We define $\alpha_{j}$ to be the left invariant one-form on $U(2)$ defined by $\alpha_{j}\left(x_{k}\right)=\delta_{j k}$. We note that

$$
J \alpha_{1} \wedge \alpha_{2}=\alpha_{3} \wedge \alpha_{4}, J \alpha_{1} \wedge \alpha_{3}=-\alpha_{2} \wedge \alpha_{4}, J \alpha_{2} \wedge \alpha_{3}=\alpha_{1} \wedge \alpha_{4}
$$

and

$$
J \alpha_{1} \wedge \alpha_{4}=-\alpha_{2} \wedge \alpha_{3}, J \alpha_{2} \wedge \alpha_{4}=\alpha_{1} \wedge \alpha_{3}, J \alpha_{3} \wedge \alpha_{4}=-\alpha_{1} \wedge \alpha_{2} .
$$

From this we note

Lemma 6 We have $J^{2}=-I$ on each space $\wedge^{2} T^{*}(U(2))_{u}$. Furthermore a basis of the eigenspace for $i$ in $\wedge^{2} T^{*}(U(2))_{u} \otimes \mathbb{C}$ is

$$
\mathcal{B}_{i}=\left\{\alpha_{1} \wedge \alpha_{4}+i \alpha_{2} \wedge \alpha_{3}, \alpha_{2} \wedge \alpha_{4}-i \alpha_{1} \wedge \alpha_{3}, \alpha_{3} \wedge \alpha_{4}+i \alpha_{1} \wedge \alpha_{2}\right\}
$$

a basis for the eigenspace $-i$ is

$$
\mathcal{B}_{-i}=\left\{\alpha_{1} \wedge \alpha_{4}-i \alpha_{2} \wedge \alpha_{3}, \alpha_{2} \wedge \alpha_{4}+i \alpha_{1} \wedge \alpha_{3}, \alpha_{3} \wedge \alpha_{4}-i \alpha_{1} \wedge \alpha_{2}\right\} .
$$

If $\mu \in \mathcal{B}_{i}$ and $\nu \in \mathcal{B}_{-i}$, then $\mu \wedge \nu=0$.

We look upon $J$ as an operator on $\Omega^{2}(U(2))_{\mathbb{C}}$. Since $J$ preserves the real vector space $\Omega^{2}(U(2))$ we get a decomposition

$$
\Omega^{2}(U(2))_{\mathbb{C}}=\Omega^{2}(U(2))_{i} \oplus \Omega^{2}(U(2))_{-i}
$$

with $J_{\mid \Omega^{2}(U(2))_{ \pm i}}= \pm i$. If $\omega \in \Omega^{2}(U(2))_{\mathbb{C}}$ then we denote by $\bar{\omega}$ the complex conjugate of $\omega$ relative to the real space $\Omega^{2}(U(2))$. We note

Lemma 7 With the notation above, we have

$$
\Omega^{2}(U(2))_{ \pm i}=\bigoplus_{\mu \in \mathcal{B}_{ \pm i}} C^{\infty}(U(2), \mathbb{C}) \mu .
$$


We now calculate the exterior derivatives of the $\alpha_{j}$. We observe that if $\alpha$ is a left invariant element of $\Omega^{1}(U(2))$, then

$$
d \alpha(x, y)=-\alpha([x, y])
$$

for $x, y \in \operatorname{Lie}(U(2))$. This implies that

$$
d \alpha_{1}=-2 \alpha_{2} \wedge \alpha_{3}, d \alpha_{2}=2 \alpha_{1} \wedge \alpha_{3}, d \alpha_{3}=-2 \alpha_{1} \wedge \alpha_{2} .
$$

We also note that $d \alpha_{4}=0$.

We denote by $\chi_{k}$ the character of $S U(2) \times S^{1}$ given by $\chi_{k}(u, z)=z^{k}$. We denote by $\pi$ the covering map $\pi: S U(2) \times S^{1} \rightarrow U(2)$ given by $\pi(u, z)=u z$. Then we have

Lemma 8 We have

$$
\left(\operatorname{ker} d_{\mid \Omega^{2}(U(2))_{\mathbb{C}}}\right)_{k, k, r}=\chi_{r} d C^{\infty}(U(2), \mathbb{C})_{k, k, 0} \wedge \alpha_{4}
$$

which is defined on $U(2)$ if $r \equiv k \bmod 2$.

Proof. We note that if $f \in C^{\infty}(U(2), \mathbb{C})$, then $d f=\left(x_{4} f\right) \alpha_{4}+\nu$ with $\nu=\sum_{j}\left(x_{j} f\right) \alpha_{j}$. Thus if $d f \wedge \alpha_{4}=0$ then $\nu=0$. If $\nu=0$ then $\pi^{*} f(u, z)=$ $\pi^{*} f(I, z)$. A function in $C^{\infty}(U(2), \mathbb{C})_{k, k, 0}$ with this property exists if and only if $k=0$. It is also clear that $\chi_{r} d C^{\infty}(U(2), \mathbb{C})_{k, k, 0} \wedge \alpha_{4}$ is contained in ker $d$. Thus, since each of the isotypic components of ker $d_{\mid \Omega^{2}(U(2))_{\mathbb{C}}}$ is irreducible and we have accounted for all of them by Lemma 4, the result follows.

We denote by Maxw the space of complex solutions to the Maxwell equations (as described in the previous section). Then Maxw is a closed subspace of $\Omega^{2}(U(2))_{\mathbb{C}}$ yielding a smooth Fréchet representation of $G$ of moderate growth under the action $\mu(g) \omega=\left(g^{-1}\right)^{*} \omega$. We also note that $J$ preserves Maxw and commutes with the action of $G$. This implies that Maxw $=\mathrm{Maxw}_{i}$ $\oplus \operatorname{Maxw}_{-i}$ (corresponding to the $i$ and $-i$ eigenspaces of $J$ on Maxw). We also note

Lemma $9 \operatorname{Maxw}_{ \pm i}=\left\{\omega \in \Omega^{2}(U(2))_{\mathbb{C}} \mid J \omega= \pm i \omega\right.$ and $\left.d \omega=0\right\}$

We can now eliminate some isotypic components of Maxw.

Lemma $10 \operatorname{Maxw}_{k, k, r}=0$ for all $k \in \mathbb{Z}_{\geq 0}$ and all $r \in \mathbb{Z}$. 
Proof. If $\omega \in \operatorname{Maxw}_{k, k, r}$, then since

$$
\operatorname{Maxw}_{k, k, r}=\operatorname{Maxw}_{k, k, r} \cap \operatorname{Maxw}_{i} \oplus \operatorname{Maxw}_{k, k, r} \cap \operatorname{Maxw}_{-i}
$$

with each of the summands $K$-invariant and since each isotypic component is irreducible we see that $J \omega=i \omega$ of $J \omega=-i \omega$. In either case Lemma 6 implies that $\omega$ is not an element of $\Omega^{1}(U(2))_{\mathbb{C}} \wedge \alpha_{4}$. But Lemma 7 implies that it must be of that form. This implies that $\omega=0$.

We are finally ready to give the isotypic components of Maxw.

Theorem $11 \mathrm{Maxw}_{p, q, r}$ is nonzero if and only if $(p, q, r)$ is in one the following forms

$$
(k+2, k, k+2),(k+2, k,-(k+2)),(k, k+2, k+2),(k, k+2,-(k+2)) .
$$

If it is nonzero it is irreducible. Moreover, $\mathrm{Maxw}_{k+2, k, k+2}$ and $\mathrm{Maxw}_{k, k+2,-k-2}$ are contained in $\mathrm{Maxw}_{i}$ and $\mathrm{Maxw}_{k+2, k,-k-2}$ and $\mathrm{Maxw}_{k, k+2, k+2}$ are contained in $\mathrm{Maxw}_{-i}$.

The proof will occupy the rest of the section. If $x \in \mathfrak{s l}(2, \mathbb{C})$ with $x=$ $v+i w, v, w \in \mathfrak{s} u(2)$, then we define the left invariant vector field $x^{L} f(u)=$ $\frac{d}{d t}\left(f\left(u e^{t v}\right)+i f\left(u e^{t w}\right)\right)_{\mid t=0}$ and $x^{R} f(u)=\frac{d}{d t}\left(f\left(e^{-t v} u\right)+i f\left(e^{-t w} v\right)\right)_{\mid t=0}$. We will think of these vector fields as being on $S U(2)$ or $S U(2) /\{ \pm I\}=U(2) / S^{1} I=$ $P S U(2)$. We set $e=\frac{1}{2}\left(x_{2}-i x_{3}\right), f=-\frac{1}{2}\left(x_{2}+i x_{3}\right)$ and $h=-i x_{1}$. Then $e, f, h$ form the standard basis of $\mathfrak{s l}(2, \mathbb{C})$.

Let $\xi_{e}^{L}, \xi_{f}^{L}, \xi_{h}^{L}$ (respectively, $\xi_{e}^{R}, \xi_{f}^{R}, \xi_{h}^{R}$ ) be the left (resp. right) invariant one-forms on $P S U(2)$ that form a dual basis to $e^{L}, f^{L}, h^{L}$ (respectively, $\left.e^{R}, f^{R}, h^{R}\right)$. Let $p: U(2) \rightarrow P S U(2)$ be the obvious quotient homomorphism and let $\alpha_{b}^{a}=p^{*} \xi_{b}^{a}$ for $a=L$ or $R$ and $b=e, f$ or $h$. Now $\left((u, v)^{-1}\right)^{*} \alpha_{b}^{L}\left(x^{L}\right)=$ $\alpha_{b}^{L}\left(\operatorname{Ad}(v)^{-1} x^{L}\right)$ and $\left((u, v)^{-1}\right)^{*} \alpha_{b}^{R}\left(x^{R}\right)=\alpha_{b}^{R}\left(\operatorname{Ad}(u)^{-1} x^{R}\right)$. Thus, if

$$
g=\left(\left[\begin{array}{cc}
z & 0 \\
0 & z^{-1}
\end{array}\right],\left[\begin{array}{cc}
w & 0 \\
0 & w^{-1}
\end{array}\right]\right)
$$

with $z, w \in S^{1}$, then

$$
\left(g^{-1}\right)^{*} \alpha_{e}^{L}=w^{-2} \alpha_{e}^{L},\left(g^{-1}\right)^{*} \alpha_{f}^{L}=w^{2} \alpha_{f}^{L},\left(g^{-1}\right)^{*} \alpha_{h}^{L}=\alpha_{h}^{L}
$$

and

$$
\left(g^{-1}\right)^{*} \alpha_{e}^{R}=u^{-2} \alpha_{e}^{R},\left(g^{-1}\right)^{*} \alpha_{f}^{R}=u^{2} \alpha_{f}^{R},\left(g^{-1}\right)^{*} \alpha_{h}^{R}=\alpha_{h}^{R} .
$$


This implies that

$$
\operatorname{Span}_{\mathbb{C}}\left(\alpha_{e}^{L}, \alpha_{f}^{L}, \alpha_{h}^{L}\right)=\Omega^{1}(U(2))_{0,2,0}
$$

and

$$
\operatorname{Span}_{\mathbb{C}}\left(\alpha_{e}^{R}, \alpha_{f}^{R}, \alpha_{h}^{R}\right)=\Omega^{1}(U(2))_{2,0,0} .
$$

Also relative to the positive root system $g \rightarrow u^{2}, g \rightarrow w^{2}$ the highest weight space of $\Omega^{1}(U(2))_{0,2,0}$ in $\mathbb{C} \alpha_{f}^{L}$ and that of $\Omega^{1}(U(2))_{2,0,0}$ is $\mathbb{C} \alpha_{f}^{R}$.

We can now describe highest weight vectors for the isotypic components $\Omega^{1}(U(2))_{k, k+2, l}$ and $\Omega^{1}(U(2))_{k+2, k, l}$. Let $e_{1}$ and $e_{2}$ be the standard basis of $\mathbb{C}^{2}$. Fix a $U(2)$ invariant inner product $\langle\ldots, \ldots\rangle$ on each space $S^{k}\left(\mathbb{C}^{2}\right)$. Define

$$
\phi_{k}(u)=\left\langle S^{k}(u) e_{1}^{k}, e_{2}^{k}\right\rangle .
$$

Then $\phi_{k}$ is a highest weight vector for $C^{\infty}(U(2))_{k, k, k}=\Omega^{0}(U(2))_{k, k, k}$. Also we define $\chi_{l}(u, z)=z^{l}$ for $u \in S U(2)$ and $z \in S^{1}$. We note that if $l \equiv k \bmod 2$, then

$$
\psi_{k, l}(u z)=z^{l-k} \phi_{k}(u z)=\chi_{l-k}(u, z) \phi_{k}(u z)
$$

is defined and is a highest weight vector for $\Omega^{0}(U(2))_{k, k, l}$. This implies that $\psi_{k, l} \alpha_{f}^{L}$ is a highest weight vector for $\Omega^{1}(U(2))_{k, k+2, l}$ and $\psi_{k, l} \alpha_{f}^{R}$ is a highest weight vector for $\Omega^{1}(U(2))_{k+2, k, l}$. We have shown that Maxw is multiplicity free and Maxw $=\operatorname{Maxw}_{i} \oplus \operatorname{Maxw}_{-i}$. We have also proved that $\operatorname{Maxw}_{k, k+2, l}=$ $d \Omega^{1}(U(2))_{k, k+2, l}$ and $\operatorname{Maxw}_{k+2, k, l}=d \Omega^{1}(U(2))_{k+2, k, l}$. We have proved that

(1) $\operatorname{Maxw}_{k, k+2, l} \neq 0$ if and only if $J d \psi_{k, l} \alpha_{f}^{L}=\lambda J \psi_{k, l} \alpha_{f}^{L}$ with $\lambda \in\{ \pm i\}$ and $\operatorname{Maxw}_{k+2, k, l} \neq 0$ if and only if $J d \psi_{k, l} \alpha_{f}^{R}=\lambda J \psi_{k, l} \alpha_{f}^{R}$ with $\lambda \in\{ \pm i\}$.

We are now left with a computation. One checks as above (using $d \alpha_{f}^{L}\left(X^{L}, Y^{L}\right)=$ $\left.-\alpha_{f}^{L}\left([X, Y]^{L}\right)\right)$

$$
d \alpha_{f}^{L}=2 \alpha_{h}^{L} \wedge \alpha_{f}^{L}
$$

we therefore have

$$
d\left(\psi_{k, l} \alpha_{f}^{L}\right)=i l \psi_{k, l} \alpha_{4} \wedge \alpha_{f}^{L}+(k+2) \psi_{k, l} \alpha_{h}^{L} \wedge \alpha_{f}^{L} .
$$

We consider $i l \alpha_{4} \wedge \alpha_{f}^{L}+(k+2) \alpha_{h}^{L} \wedge \alpha_{f}^{L}$. We observe that $\alpha_{h}^{L}=i \alpha_{1}$ and $\alpha_{f}^{L}=-\left(\alpha_{2}-i \alpha_{3}\right)$. Thus (using the calculations leading to Lemma 6) the right-hand side of the equation above is equal to

$$
l i \alpha_{4} \wedge \alpha_{2}-l \alpha_{4} \wedge \alpha_{3}+i(k+2) \alpha_{1} \wedge\left(\alpha_{2}-i \alpha_{3}\right)
$$


which equals

$$
\left(-l i \alpha_{2} \wedge \alpha_{4}+(k+2) J \alpha_{2} \wedge \alpha_{4}\right)+\left(-l \alpha_{1} \wedge \alpha_{4}-i(k+2) J \alpha_{1} \wedge \alpha_{4} .\right.
$$

We therefore see that if $l>0$ then this expression is an element of $\Omega^{2}(U(2))_{-i}+$ $(l-k-2)\left(-i \alpha_{2} \wedge \alpha_{4}-(l-k-2) \alpha_{1} \wedge \alpha_{4}\right)$. If $l \leq 0$ then it is an element of $\Omega^{2}(U(2))_{i}+(l+k+2)\left(-i \alpha_{2} \wedge \alpha_{4}-(l+k+2) \alpha_{1} \wedge \alpha_{4}\right)$. Thus we have

(2) $\operatorname{Maxw}_{k, k+2, l} \neq 0$ only if $l=k+2$ or $l=-(k+2)$. Furthermore, $\operatorname{Maxw}_{k, k+2, k+2} \subset \mathrm{Maxw}_{-i}$ and $\operatorname{Maxw}_{k, k+2,-(k+2)} \subset \mathrm{Maxw}_{i}$.

We note that everything that we have done could have been done with right-invariant vector fields to complete the proof of the theorem. However we will proceed in a different way. Let $\eta: U(2) \rightarrow U(2)$ be defined by $\eta(u)=u^{-1}$. Then for $x \in \mathfrak{u}(2)$, we have $d \eta_{u}\left(x_{u}^{L}\right)=x_{u^{-1}}^{R}$. This implies that

$$
\left\langle d \eta_{u}\left(x_{u}^{L}\right), d \eta_{u}\left(x_{u}^{L}\right)\right\rangle_{u^{-1}}=\left\langle x_{u^{-1}}^{R}, x_{u^{-1}}^{R}\right\rangle_{u^{-1}}=-\operatorname{det} x
$$

since

$$
\left\langle x_{u^{-1}}^{R}, x_{u^{-1}}^{R}\right\rangle_{u^{-1}}=\left\langle d R\left(u^{-1}\right)_{I}\left(x_{I}^{R}\right), d R\left(u^{-1}\right)_{I}\left(x_{I}^{R}\right)\right\rangle_{u^{-1}}=\left\langle x_{I}^{R}, x_{I}^{R}\right\rangle_{I} .
$$

This proves that $\eta$ is an isometry. It also implies that

$$
\left(\eta^{*} \alpha_{f}^{R}\right)_{u}\left(x_{u}^{L}\right)=\left(\alpha_{f}^{R}\right)_{u^{-1}}\left(d \eta_{u}\left(x_{u}^{L}\right)\right)=\left(\alpha_{f}^{R}\right)_{u^{-1}}\left(x_{u^{-1}}^{R}\right) .
$$

Hence $\eta^{*} \alpha_{f}^{R}=\alpha_{f}^{L}$. Now $\eta^{*} d\left(\psi_{k, l} \alpha_{f}^{R}\right) \in \Omega^{2}(U(2))_{k, k+2,-l}$ since $\eta^{*} \phi_{k}$ is a highest weight vector for $C^{\infty}(U(2))_{k, k,-k}$. We also note that $\eta^{*} \gamma=\gamma$. Thus $\eta^{*}$ Maxw $=$ Maxw. Hence, if $d\left(\psi_{k, l} \alpha_{f}^{R}\right) \in \operatorname{Maxw}_{k+2, k, l}$, then $\eta^{*} d\left(\psi_{k, l} \alpha_{f}^{R}\right) \in$ $\operatorname{Maxw}_{k, k+2,-l}$. So, if $l>0$ then, we must have $l=k+2$ and if $l \leq 0$, then $l=-k-2$. Since $\eta^{*}$ commutes with $J$ the last assertion also follows.

Remark 12 We have

$$
\begin{gathered}
\eta^{*} \operatorname{Maxw}_{k+2, k, k+2}=\operatorname{Maxw}_{k, k+2,-k-2}, \\
\eta^{*} \operatorname{Maxw}_{k+2, k,-k-2}=\operatorname{Maxw}_{k, k+2, k+2} \text { and }\left(\eta^{*}\right)^{2}=I .
\end{gathered}
$$




\section{The Hermitian form}

We retain the notation of the previous sections.

We note that

$$
H_{3}(U(2), \mathbb{R})=\mathbb{R}
$$

The form $\nu=\alpha_{1} \wedge \alpha_{2} \wedge \alpha_{3}$ restricted to $S U(2)$ satisfies

$$
\int_{S U(2)} \nu=2 \pi^{2}
$$

and $d \nu=0$. This implies that the class of $S U(2)$ in the third homology over $\mathbb{R}$ is a basis. (In fact it is well known that this is true over $\mathbb{Z}$ ). We note that this implies, in particular, that if $\omega \in \Omega^{3}(U(3))_{\mathbb{C}}$ satisfies $d \omega=0$ then if $M$ is a compact submanifold such that there exists a smooth family of diffeomorphisms of $U(2), \Phi_{t}$ such that $\Phi_{0}=I$ and $\Phi_{1}(S U(2))=M$, then

$$
\int_{S U(2)} \omega=\int_{M} \omega .
$$

In particular we have (since $\mathrm{U}(2,2)$ is connected),

Lemma 13 If $g \in U(2,2)$ and if $\omega \in \Omega^{3}(U(3))_{\mathbb{C}}$ satisfies $d \omega=0$, then $\int_{S U(2)} \omega=\int_{g S U(2)} \omega=\int_{S U(2)} g^{*} \omega$.

We will apply this observation to define $U(2,2)$ invariant sesquilinear forms on the spaces $\mathrm{Maxw}_{ \pm i}$.

Lemma 14 Let $\alpha \in \Omega^{1}(U(2))_{\mathbb{C}}$ and $\omega \in \Omega^{2}(U(2))_{\mathbb{C}}$ be such that $\omega, d \alpha \in$ $\operatorname{Maxw}_{\mathrm{i}}$ (resp. $\left.\mathrm{Maxw}_{-i}\right)$. Then $d(\alpha \wedge \bar{\omega})=0$.

Proof. If $d \alpha \in \operatorname{Maxw}_{i}$ then $\bar{\omega} \in \mathrm{Maxw}_{-i}$. We note that Maxwell's equations imply that $d \bar{\omega}=0$. Hence $d(\alpha \wedge \bar{\omega})=d \alpha \wedge \bar{\omega}$ and Lemma 6 implies that $\mathrm{Maxw}_{i} \wedge \mathrm{Maxw}_{-i}=0$. Obviously the same argument works for $-i$.

Proposition 15 If $\mu, \omega \in \operatorname{Maxw}_{i}$ (or $\mathrm{Maxw}_{-i}$ ), there exists $\alpha \in \Omega^{1}(U(2))_{\mathbb{C}}$ such that $d \alpha=\omega$. The expression

$$
\int_{S U(2)} \alpha \wedge \bar{\mu}
$$

depends only on $\omega$ and $\mu$ (and not on the choice of $\alpha$ ). Furthermore, the integral defines a Hermitian form $\langle\omega, \mu\rangle$ on $\operatorname{Maxw}_{i}($ or Maxw-i) that satisfies $\left\langle g^{*} \omega, g^{*} \mu\right\rangle=\langle\omega, \mu\rangle$ for all $g \in U(2,2)$. 
Proof. Suppose that $\beta \in \Omega^{1}(U(2))_{\mathbb{C}}$ is such that $d \beta=0$ and $\int_{S^{1} I} \beta=0$. Then since $H_{1}(U(2), \mathbb{C})$ is spanned by the class of $S^{1} I$, de Rham's theorem implies that there exists $f \in C^{\infty}(U(2), \mathbb{C})$ such that $d f=\beta$. Set $\iota(\beta)=$ $\int_{S^{1} I} \beta$. We note that

$$
\iota\left(\alpha_{1}\right)=2 \pi .
$$

We also note that if $\nu \in \Omega^{2}(U(2))$ then $\left(\alpha_{1} \wedge \nu\right)_{\mid S U(2)}=0$.

We observed that if $\omega \in \mathrm{Maxw}_{ \pm i}$, then there exists $\alpha \in \Omega^{1}(U(2))_{\mathbb{C}}$ such that $d \alpha=\omega$. If $d \beta=\omega$ then $d(\beta-\alpha)=0$ and $\int_{S^{1} I}\left(\beta-\alpha-\frac{\iota(\beta-\alpha)}{2 \pi} \alpha_{1}\right)=0$ so $\beta-\alpha-\frac{\iota(\beta-\alpha)}{2 \pi} \alpha_{1}=-d f$ with $f \in C^{\infty}(U(2), \mathbb{C})$. This implies that

$$
\begin{gathered}
\int_{S U(2)} \alpha \wedge \bar{\mu}-\int_{S U(2)} \beta \wedge \bar{\mu}=\int_{S U(2)}\left(d f-\frac{\iota(\beta-\alpha)}{2 \pi} \alpha_{1}\right) \wedge \bar{\mu} \\
=\int_{S U(2)} d(f \bar{\mu})-\frac{\iota(\beta-\alpha)}{2 \pi} \int_{S U(2)} \alpha_{1} \wedge \bar{\mu}=0 .
\end{gathered}
$$

Both of the integrals are 0 . We next observe that $\mu=d \xi$. We have

$$
\begin{aligned}
& \overline{\langle\mu, \omega\rangle}-\langle\omega, \mu\rangle=\int_{S U(2)}(\bar{\xi} \wedge d \alpha-\alpha \wedge d \bar{\xi}) \\
& =\int_{S U(2)}(\bar{\xi} \wedge d \alpha-d \bar{\xi} \wedge \alpha)=-\int_{S U(2)} d(\bar{\xi} \wedge \alpha)=0 .
\end{aligned}
$$

We are left with the proof of $U(2,2)$ invariance. We will concentrate on $\operatorname{Maxw}_{i}$. We note that

$$
\int_{S^{1} I} \alpha_{1}=2 \pi
$$

Let $g \in U(2,2)$. If $\omega, \mu \in \operatorname{Maxw}_{i}$ and $\alpha \in \Omega^{1}(U(2))_{\mathbb{C}}$ satisfies $d \alpha=\omega$, then $d g^{*} \alpha=g^{*} \omega$. Thus

$$
\left\langle g^{*} \omega, g^{*} \mu\right\rangle=\int_{S U(2)} g^{*} \alpha \wedge g^{*} \bar{\mu}=\int_{S U(2)} \alpha \wedge \bar{\mu}=\langle\omega, \mu\rangle
$$

by Lemma 13 .

We will now calculate $\langle\ldots, \ldots\rangle$ on each of the isotypic components of Maxw. We set $\alpha_{k, k+2, l}=\psi_{k, l} \alpha_{f}^{L}$ as in the previous section. If $l=k+2$ or $-(k+2)$, then $\omega_{k, k+2, l}=d \alpha_{k, k+2, l}$ is a highest weight vector for $\operatorname{Maxw}_{k, k+2, l}$. 
Lemma 16 If $l= \pm(k+2)$ then $\left\langle\omega_{k, k+2, l}, \omega_{k, k+2, l}\right\rangle=-\frac{4 k+8}{k+1} \pi^{2}$.

Proof. We have seen in formula (2) in the previous section that (in the notation therein)

$$
\omega_{k, k+2, l}=i l \psi_{k, l} \alpha_{4} \wedge \alpha_{f}^{L}+(k+2) \psi_{k, l} \alpha_{h}^{L} \wedge \alpha_{f}^{L} .
$$

Thus since $\alpha_{4 \mid S U(2)}=0$ we have

$$
\alpha_{k, k+2, l} \wedge{\overline{\omega_{k, k+2, l} \mid S U(2)}}=(k+2)\left|\psi_{k, l}\right|^{2} \alpha_{f}^{L} \wedge \overline{\alpha_{h}^{L} \wedge \alpha_{f}^{L}} .
$$

Using the expressions for $\alpha_{h}^{L}$ and $\alpha_{f}^{L}$ one sees that

$$
\alpha_{f}^{L} \wedge \overline{\alpha_{h}^{L} \wedge \alpha_{f \mid S U(2)}^{L}}=-2 \alpha_{1} \wedge \alpha_{2} \wedge \alpha_{3 \mid S U(2)} .
$$

Normalized invariant measure on $\mathrm{SU}(2)$ is $\mu=\frac{1}{2 \pi^{2}} \alpha_{1} \wedge \alpha_{2} \wedge \alpha_{3 \mid S U(2)}$. On $S^{k}\left(\mathbb{C}^{2}\right)$ we put the tensor-product inner product. Thus $e_{1}^{k}$ and $e_{2}^{k}$ are unit vectors. Also

$$
\left|\psi_{k, l}(u)\right|^{2}=\left\langle S^{k}(u) e_{1}^{k}, e_{2}^{k}\right\rangle \overline{\left\langle S^{k}(u) e_{1}^{k}, e_{2}^{k}\right\rangle} .
$$

The Schur orthogonality relations imply that

$$
\int_{S U(2)}\left|\psi_{k, l}(u)\right|^{2} \mu=\frac{1}{k+1} .
$$

\section{Four unitary ladder representations of $S U(2,2)$}

We will consider $U(2,2)$ in the usual form that is if $I_{2,2}=\left[\begin{array}{cc}I & 0 \\ 0 & -I\end{array}\right]$, then (as in Section 2)

$$
G_{1}=\left\{g \in S L(4, \mathbb{C}) \mid g I_{2,2} g^{*}=I_{2,2}\right\} .
$$

In this form $K$ is the subgroup of block diagonal matrices. $\mathfrak{g}=\operatorname{Lie}(U(2,2))_{\mathbb{C}}=$ $M_{4}(\mathbb{C})$. We set

$$
\mathfrak{p}^{+}=\left\{\left[\begin{array}{cc}
0 & X \\
0 & 0
\end{array}\right] \mid X \in M_{2}(\mathbb{C})\right\}
$$


and

$$
\mathfrak{p}^{-}=\left\{\left[\begin{array}{cc}
0 & 0 \\
Y & 0
\end{array}\right] \mid Y \in M_{2}(\mathbb{C})\right\} .
$$

Then $\mathfrak{g}=\operatorname{Lie}(K)_{\mathbb{C}} \oplus \mathfrak{p}$ (here $\mathfrak{p}$ is the orthogonal complement of $\operatorname{Lie}(K)_{\mathbb{C}}$ relative to the trace form) and as a $K$-module $\mathfrak{p}=\mathfrak{p}^{+} \oplus \mathfrak{p}^{-}$. We leave it to the reader to check

Lemma 17 As a representation of $K$ (under the adjoint action) $\mathfrak{p}^{+} \cong F^{1,1,-1}$ and $\mathfrak{p}^{-} \cong F^{1,1,1}$ and

$$
\wedge^{2}\left(\mathfrak{p}^{+}\right)^{*} \cong F^{2,0,2} \oplus F^{0,2,2}
$$

and

$$
\wedge^{2}\left(\mathfrak{p}^{-}\right)^{*} \cong F^{2,0,-2} \oplus F^{0,2,-2}
$$

Let $\pi(g) \omega=\left(g^{-1}\right)^{*} \omega$ for $\omega \in$ Maxw. Then we already observed that with the $C^{\infty}$-topology, $(\pi$, Maxw) is an admissible smooth Fréchet representation of moderate growth. We set $\mathrm{Maxw}_{K}$ equal to the space of $K$-finite vectors of Maxw and we will use module notation for the action of $\mathfrak{g}=\operatorname{Lie}(U(2,2))_{\mathbb{C}}=$ $M_{4}(\mathbb{C})$ on $\operatorname{Maxw}_{K}$, thereby having an admissible finitely generated $(\mathfrak{g}, K)$ module.

Lemma $18 \mathfrak{p}^{+}$annihilates $\operatorname{Maxw}_{2,0,2}$ and $\operatorname{Maxw}_{0,2,2}, \mathfrak{p}^{-}$annihilates $\mathrm{Maxw}_{2,0,-2}$ and $\mathrm{Maxw}_{0,2,-2}$.

Proof. Using the Clebsch-Gordon formula we have

$$
\begin{gathered}
F^{1,1,-1} \otimes F^{2,0,2} \cong F^{3,1,1} \oplus F^{1,1,1}, \\
F^{1,1,-1} \otimes F^{0,2,2} \cong F^{1,3,1} \oplus F^{1,1,1}, \\
F^{1,1,1} \otimes F^{2,0,-2} \cong F^{3,1,-1} \oplus F^{1,1,-1}, \\
F^{1,1,1} \otimes F^{0,2,-2} \cong F^{1,3,-1} \oplus F^{1,1,-1} .
\end{gathered}
$$

Theorem 11 implies that none of the $K$-types on the right of these equations occurs in Maxw.

We set

$$
\left(\operatorname{Maxw}_{2,0}^{+}\right)_{K}=\sum_{k \geq 0} \operatorname{Maxw}_{k+2, k, k+2},\left(\operatorname{Maxw}_{0,2}^{+}\right)_{K}=\sum_{k \geq 0} \operatorname{Maxw}_{k, k+2, k+2}
$$




$$
\left(\operatorname{Maxw}_{2,0}^{-}\right)_{K}=\sum_{k \geq 0} \operatorname{Maxw}_{k+2, k,-k-2} \operatorname{and}\left(\operatorname{Maxw}_{0,2}^{-}\right)_{K}=\sum_{k \geq 0} \operatorname{Maxw}_{k, k+2,-k-2} .
$$

We will drop the sub- $K$ for the completions of these spaces. We note Theorem 11 implies that each of these spaces is totally contained in $\mathrm{Maxw}_{i}$ or $\mathrm{Maxw}_{-i}$. This implies that the Hermitian form, $\langle\ldots, \ldots\rangle$ from the previous section is defined on each of these spaces.

We have

Theorem 19 Each of the spaces $\mathrm{Maxw}_{2,0}^{+}, \mathrm{Maxw}_{2,0}^{-}, \mathrm{Maxw}_{0,2}^{+}, \mathrm{Maxw}_{0,2}^{-}$is an invariant irreducible subspace for $\pi$. Furthermore, the form $\langle\ldots, \ldots\rangle$ is positive definite on $\mathrm{Maxw}_{2,0}^{+}, \mathrm{Maxw}_{2,0}^{-}$and negative definite on $\mathrm{Maxw}_{0.2}^{+}$, $\mathrm{Maxw}_{0,2}^{-}$.

Proof. We consider $\mathrm{Maxw}_{2,0}^{+}$. We note that using the Clebsch-Gordan formula as above we have

$$
\begin{gathered}
\mathfrak{p}^{-} \operatorname{Maxw}_{k+2, k, k+2} \subset \operatorname{Maxw}_{k+3, k+1, k+3}+\operatorname{Maxw}_{k+3, k-1, k+3} \\
+\operatorname{Maxw}_{k+1, k+2, k+3}+\operatorname{Maxw}_{k+1, k-1, k+3}
\end{gathered}
$$

and

$$
\begin{gathered}
\mathfrak{p}^{+} \operatorname{Maxw}_{k+2, k, k+2} \subset \operatorname{Maxw}_{k+3, k+1, k+1}+\operatorname{Maxw}_{k+3, k-1, k+1} \\
+\operatorname{Maxw}_{k+1, k+1, k+1}+\operatorname{Maxw}_{k+1, k-1, k+1} .
\end{gathered}
$$

Using the form of the $K$-types of Maxw we see that

$$
\mathfrak{p}^{-} \operatorname{Maxw}_{k+2, k, k+2} \subset \operatorname{Maxw}_{k+3, k+1, k+3}
$$

and

$$
\mathfrak{p}^{+} \operatorname{Maxw}_{k+2, k, k+2} \subset \operatorname{Maxw}_{k+1, k-1, k+1} .
$$

This implies that $\left(\mathrm{Maxw}_{2,0}^{+}\right)_{\mathrm{K}}$ is a $(\mathfrak{g}, K)$-submodule of Maxw. Hence $\mathrm{Maxw}_{2,0}^{+}$ is $G$-invariant. The same argument proves

$$
\begin{aligned}
\mathfrak{p}^{-} \operatorname{Maxw}_{k, k+2, k+2} & \subset \operatorname{Maxw}_{k+1, k+3, k+3}, \\
\mathfrak{p}^{+} \operatorname{Maxw}_{k, k+2, k+2} & \subset \operatorname{Maxw}_{k-1, k+1, k+1}, \\
\mathfrak{p}^{+} \operatorname{Maxw}_{k+2, k,-(k+2)} & \subset \operatorname{Maxw}_{k+3, k+1,-(k+3)} \\
\mathfrak{p}^{-} \operatorname{Maxw}_{k+2, k,-(k+2)} & \subset \operatorname{Maxw}_{k+1, k-1,-(k+1)}
\end{aligned}
$$


and

$$
\begin{aligned}
\mathfrak{p}^{+} \operatorname{Maxw}_{k, k+2,-(k+2)} & \subset \operatorname{Maxw}_{k+1, k+2,-(k+3)} \\
\mathfrak{p}^{-} \operatorname{Maxw}_{k, k+2,-(k+2)} & \subset \operatorname{Maxw}_{k-1, k+1,-(k+1)} .
\end{aligned}
$$

This proves the $G$-invariance of the indicated spaces. We next note that since the multiplicities of the $K$-types of Maxw are all one, the Hermitian form $\langle\ldots, \ldots\rangle$ restricted to each space $\operatorname{Maxw}_{k, l, m}$ is 0 , positive definite or negative definite. Thus Lemma 16 implies that the form is negative definite on $\left(\operatorname{Maxw}_{0,2}^{+}\right)_{K}$ and $\left(\operatorname{Maxw}_{0,2}^{-}\right)_{K}$. Recall that $\eta^{*}\left(\operatorname{Maxw}_{0,2}^{+}\right)_{K}=\left(\operatorname{Maxw}_{2,0}^{-}\right)_{K}$ and $\left.\eta^{*} \operatorname{Maxw}_{0,2}^{-}\right)_{K}=\left(\operatorname{Maxw}_{2,0}^{+}\right)_{K}$. Furthermore $\eta$ is orientation reversing on $S U(2)$, thus the form on $\left(\operatorname{Maxw}_{2,0}^{+}\right)_{K}$ and $\left(\operatorname{Maxw}_{2,0}^{-}\right)_{K}$ is positive definite. If one of the representations were not irreducible, then it would have a finitedimensional invariant subspace (by the above formulae). Since these representations are all unitarizable and do not contain one-dimensional invariant subspaces the representations are all irreducible.

Remark 20 The proof above implies that the last six inclusions above are all equalities.

We set $\pi_{i, j}^{\varepsilon}$ equal to the action of $G$ on $\operatorname{Maxw}_{i, j}^{\varepsilon}$ for $\varepsilon=+,-$ and $i, j=0,2$ or 2,0 .

Theorem 21 The representations $\pi_{i, j}^{\varepsilon}$ for $\varepsilon=+,-$ and $i, j=0,2$ or 2,0 all have trivial infinitesimal character (that is equal to the restriction to the center of $U(\mathfrak{g})$ of the augmentation homomorphism to its center). Furthermore

$$
H^{2}\left(\mathfrak{g}, K, \operatorname{Maxw}_{i, j}^{\varepsilon}\right)=\mathbb{C}, \varepsilon=+,- \text { and } i, j=0,2 \text { or } 2,0 .
$$

Proof. By the above, all four of the representations are the spaces of $C^{\infty}$ vectors of an irreducible unitary representation. Since the center of $\mathfrak{g}$ acts trivially and

$$
\operatorname{dim} \operatorname{Hom}_{K}\left(\wedge^{2} \mathfrak{p}, M\right)=1
$$

for each $M$ as in the statement, it is enough to prove that the Casimir operator corresponding to the trace form on $\mathfrak{g}$ acts by 0 on each of the representations (c.f. $[\mathrm{BW}]$ ). We will show that the center of the enveloping algebra acts correctly. We look at the case $\mathrm{Maxw}_{2,0}^{+}$and leave the other cases to the reader. By Lemma $18\left(\mathrm{Maxw}_{2,0}^{+}\right)_{K}$ is a highest weight module relative to the Weyl chamber (in $\varepsilon$ notation) $\varepsilon_{1}>\varepsilon_{2}>\varepsilon_{3}>\varepsilon_{4}$. We calculate 
the highest weight $\lambda=a \varepsilon_{1}+b \varepsilon_{2}+c \varepsilon_{3}+d \varepsilon_{4}$. The representation factors through the adjoint group so $a+b+c+d=0$. By definition of $F^{2,0,2}$ $a-b=2, c-d=0, c+d=2$. Solving the four equations yields $-2 \varepsilon_{2}+\varepsilon_{3}+\varepsilon_{4}$. $\rho$ for the chamber that we are studying is $\frac{3}{2} \varepsilon_{1}+\frac{1}{2} \varepsilon_{2}-\frac{1}{2} \varepsilon_{3}-\frac{3}{2} \varepsilon_{4}$. Thus $\lambda+\rho=\frac{3}{2} \varepsilon_{1}-\frac{3}{2} \varepsilon_{2}+\frac{1}{2} \varepsilon_{3}-\frac{1}{2} \varepsilon_{4}=\sigma \rho$ for $\sigma$ the cyclic permutation (243).

Let $\theta$ be the Cartan involution of $G_{1}$ corresponding to $K$. In light of the Vogan-Zuckerman theorem [VZ] this implies that there are four $\theta$ stable parabolic subalgebras $\mathfrak{q}_{i, j}^{\varepsilon} \varepsilon=+,-$ and $i, j=0,2$ or 2,0 , such that $\left(\operatorname{Maxw}_{i, j}^{\varepsilon}\right)_{K}$ is isomorphic with $A_{\mathfrak{q}_{i, j}^{\varepsilon}}(0)$ (c.f. [BW]).

Theorem $22 \mathfrak{q}_{2,0}^{+}$is the parabolic subalgebra

$$
\begin{gathered}
\left\{\left[x_{i j}\right] \in M_{4}(\mathbb{C}) \mid x_{21}=x_{31}=x_{41}=0\right\}, \\
\mathfrak{q}_{0,2}^{+}=\left\{\left[x_{i j}\right] \in M_{4}(\mathbb{C}) \mid x_{41}=x_{42}=x_{43}=0\right\},
\end{gathered}
$$

and $\mathfrak{q}_{0,2}^{-}=\left(\mathfrak{q}_{0,2}^{+}\right)^{T}, \mathfrak{q}_{2,0}^{-}=\left(\mathfrak{q}_{2,0}^{+}\right)^{T}$.

Proof. If $\mathfrak{q}$ is a $\theta$-stable parabolic subalgebra with nilpotent radical $\mathfrak{u}$ and if $\mathfrak{u}_{n}=\mathfrak{u} \cap \mathfrak{p}$, we set $2 \rho_{n}(\mathfrak{q})(h)=\operatorname{trad}(\mathrm{h}) \mathfrak{u}_{\mathrm{n}}$ for $h \in \mathfrak{h}$ the Cartan subalgebra of diagonal matrices. One checks that if the parabolics are given as in the theorem, then $2 \rho_{n}\left(\mathfrak{q}_{2,0}^{+}\right)$is the highest weight of $F^{2,0,2}, 2 \rho_{n}\left(\mathfrak{q}_{0,2}^{+}\right)$is that of $F^{0,2,2}, 2 \rho_{n}\left(\mathfrak{q}_{2,0}^{-}\right)$is the lowest weight of $F^{2,0,-2}$, and $2 \rho_{n}\left(\mathfrak{q}_{0,2}^{-}\right)$is the lowest weight of $F^{0,2,-2}$. Now the result follows from the main theorem (c.f. [W1]).

Remark 23 This result implies that if we consider the open orbit $X$ of $U(2,2)$ in $\mathbb{P}^{3}(\mathbb{C})$ that has no non-constant holomorphic functions, then there are two holomorphic line bundles and two anti-holomorphic line bundles such that their degree 1 smooth sheaf cohomology yields the four versions of solutions of Maxwell's equations. The relation between the two realizations is the Penrose-Twistor transform.

Another realization of these representations is in [GW]. The observation here is that $S U(2,2)$ is the quaternionic real form of $S L(4, \mathbb{C})$. Since $K \cap$ $S U(2,2)=S(U(2) \times U(2))$ there are two invariant quaternionic structures on $S U(2,2) / K \cap S U(2,2)$ and on each of two line bundles on which the methods of [GW] apply. Each yields an element of the "analytic continuation" of the 
corresponding quaternionic discrete series which is our third realization. We will allow the interested reader to check these assertions.

Finally, these representations appear in Howe duality between $U(2,2)$ and the $U(1)$ in its center. The details are worked out in [BW], VIII, 2.10-2.12 in the notation therein, and the pertinent representations are $V_{2}$ and $V_{-2}$ yielding $\left.\mathrm{Maxw}_{2,0}^{+}\right)_{K}$ and $\left.\mathrm{Maxw}_{0,2}^{+}\right)_{K}$. The other two constituents are gotten by duality.

\section{A dual pair in $P S U(2,2)$}

We continue to consider $U(2,2)$ as the group $G_{1}$ in Section 2 and $P S U(2,2)$ the quotient by the center. Let $S$ be the image of the subgroup of all matrices of the form

$$
\left[\begin{array}{ll}
a I & b I \\
\bar{b} I & \bar{a} I
\end{array}\right]
$$

with $|a|^{2}-|b|^{2}=1$. Then $S$ is isomorphic with $P S U(1,1)$. The image of the subgroup of $P S U(2,2)$ that centralizes every element of $S$ is the image of the group of all block $2 \times 2$ diagonal elements of $G_{1}, C$ which is isomorphic with $S O(3)$. These two groups form a reductive dual pair (the commutant of $C$ is $S$ ). Before we analyze this pair, we will indicate its relationship with time in Minkowski space. If we write coordinates in Section 2 as $x_{1}, x_{2} . x_{3}$ and $x_{4}=t$, then we have according to our rules

$$
(0,0,0, t) \longmapsto \frac{(1+i t)}{1-i t} I
$$

In terms of the linear fractional action of $U(2,2)$ on $U(2)$ this corresponds to

$$
\left[\begin{array}{cc}
\frac{1+i t}{\sqrt{1+t^{2}}} I & 0 \\
0 & \frac{1-i t}{\sqrt{1+t^{2}}} I
\end{array}\right] . I .
$$

That is, the time axis is the orbit of $K \cap S$ with $-I$ deleted.

As we observed, the center of $U(2,2)$ acts trivially on Maxw and thus we can consider the action of the group $C \times S$ through $C S$ on Maxw. Let $\mathcal{H}^{k}$ denote the representation of $S O(3)$ on the spherical harmonics of degree $k$. We denote by $D_{2 k}, k \in \mathbb{Z}-\{0\}$, the discrete series of $S$ ( $D_{2 k}$ has $K$-types $\left.2 k+2 \operatorname{sgn}(k) \mathbb{Z}_{\geq 0}\right)$. 
Theorem 24 As a representation of $C \times S$, $\left(\mathrm{Maxw}_{2,0}^{+}\right)_{K}$ and $\left(\mathrm{Maxw}_{0,2}^{+}\right)_{K}$ are equivalent with

$$
\bigoplus_{k \geq 1} \mathcal{H}^{k} \otimes D_{-(2 k+2)}
$$

and $\left(\mathrm{Maxw}_{2,0}^{-}\right)_{\mathrm{K}}$ and $\left(\mathrm{Maxw}_{0,2}^{-}\right)_{\mathrm{K}}$ are equivalent with

$$
\bigoplus_{k \geq 1} \mathcal{H}^{k} \otimes D_{2 k+2}
$$

Proof. By considering the $K$-types we see that each of the modules has finite $K \cap C S$-multiplicities. We will give details for $M=\left(\mathrm{Maxw}_{2,0}^{+}\right)_{K}$. Noting that $S \cap K$ is

$$
\left\{\left[\begin{array}{cc}
a I & \\
& a^{-1} I
\end{array}\right]|| a \mid=1\right\}
$$

we see that the characters that appear for $S \cap K$ on $M$ are $a^{-4}, a^{-6}, \ldots$ each with finite multiplicity. Thus, as a representation of $S, M$ is a direct sum of highest weight modules. It is therefore enough to check that the character of $M$ as a $C S \cap K$ module is correct. We note that in the formulation of the $K$-types, we can look upon $C S \cap K$ as the image of the group of all matrices

$$
\left[\begin{array}{cc}
u(y) & 0 \\
0 & u(y) x^{-2}
\end{array}\right]
$$

with $u(y)=\left[\begin{array}{cc}y & 0 \\ 0 & y^{-1}\end{array}\right]$ and $|x|$ and $|y|=1$. Set $t(x, y)$ equal to this element. Then if $C h(V)$ denotes the character of a $C S \cap K$ module and $\chi_{k}$ denotes the character of $S U(2)$ on $S^{k}\left(\mathbb{C}^{2}\right)$, we have as a formal sum

$$
C h(M)=\sum_{k=0}^{\infty} \chi_{k+2}(u(y)) \chi_{k}(u(y)) x^{-2 k-4}
$$

replacing $x$ with $x^{-1}$ and plugging in $\chi_{k}(u(y))=y^{k}\left(1+y^{-2}+\ldots+y^{-2 k}\right)$ and summing we have

$$
\frac{x^{4}\left(y^{4}-x^{2} y^{2}+y^{2}+1\right)}{\left(1-x^{2}\right)\left(y^{2}-x^{2}\right)\left(1-x^{2} y^{2}\right)} \text {. }
$$

If we multiply by $1-x^{2}$ and expand in powers of $x$, we see that the series is

$$
x^{4} \sum_{k=0}^{\infty} \chi_{2 k+2}(u(y)) x^{2 k}
$$


This implies the result in this case since the character of $D_{-2 k}$ is

$$
\frac{x^{-2 k}}{1-x^{-2}}\left(\operatorname{resp} \cdot \frac{x^{2 k}}{1+x^{2}}\right)
$$

if $k>0$ (resp. $k<0)$. The other highest weight case is exactly the same. The cases $\left(\operatorname{Maxw}_{2,0}^{-}\right)_{K}$ and $\left(\mathrm{Maxw}_{0,2}^{-}\right)_{K}$ are handled in the same way but with the powers of $x$ inverted.

\section{$9 \quad$ Plane wave solutions and (degenerate) Whittaker vectors}

Recall that a plane wave solution to Maxwell's are those in the following form

$$
\mathbf{E}=e^{i(z, x)} \mathbf{E}_{o}
$$

and

$$
\mathbf{H}=e^{i(z, x)} \mathbf{H}_{o}
$$

with $\mathbf{E}_{o}$ and $\mathbf{H}_{o}$ constant vectors, $z=\left(u_{1}, u_{2}, u_{3}, \omega\right) \in \mathbb{R}^{4}, \mathbf{u}=\left(u_{1}, u_{2}, u_{3}\right)$, $x=\left(x_{1}, x_{2}, x_{3}, t\right) \in \mathbb{R}^{4}$, and as before, $(z, x)=-\sum u_{i} x_{i}+\omega t$. To satisfy Maxwell's equations we must have $\mathbf{u} \cdot \mathbf{E}_{o}=0, \mathbf{u} \cdot \mathbf{H}_{o}=0$ and

$$
\nabla \times \mathbf{E}=\frac{\partial}{\partial t} \mathbf{H}, \nabla \times \mathbf{H}=-\frac{\partial}{\partial t} \mathbf{E} .
$$

The first implies that $\mathbf{u} \times \mathbf{E}_{o}=-\omega \mathbf{H}_{o}$ and the second $\mathbf{u} \times \mathbf{H}_{o}=\omega \mathbf{E}_{o}$. Thus, if the solution is non-constant and $\omega>0($ resp. $\omega<0) \frac{\mathbf{u}}{\|\mathbf{u}\|}, \frac{\mathbf{H}_{o}}{\left\|\mathbf{H}_{o}\right\|}, \frac{\mathbf{E}_{o}}{\left\|E_{o}\right\|}$ (resp. $\left.\frac{\mathbf{u}}{\|\mathbf{u}\|}, \frac{\mathbf{H}_{o}}{\left\|\mathbf{H}_{o}\right\|}, \frac{-\mathbf{E}_{o}}{\left\|E_{o}\right\|}\right)$ is an orthonormal basis of $\mathbb{R}^{3}$ obeying the "right-hand screw law". Putting these equations together yields $\omega^{2}=\|\mathbf{u}\|^{2}$, that is, $z$ is isotropic in $\mathbb{R}^{1,3}$. That is on the null light cone. This fits with the fact that if $\mathbf{E}, \mathbf{H}$ form a solution to Maxwell's equations, then their individual components satisfy the wave equation.

The purpose of this section is to interpret the plane wave solutions in the context of the four representations we have been studying. In the last few sections we have been emphasizing the realization of $U(2,2)$ which we have denoted by $G_{1}$. We now revert to the form $G$ since the embedding of 
Minkowski space into $U(2)$ is clearer for that realization. Recall that the embedding is implemented in two stages; we map $\left(x_{1}, x_{2}, x_{3}, x_{4}\right)$ to

$$
\left[\begin{array}{cc}
I & 0 \\
Y & I
\end{array}\right], Y=\left[\begin{array}{cc}
x_{4}+x_{3} & x_{1}+i x_{2} \\
x_{1}-i x_{2} & x_{4}-x_{3}
\end{array}\right]
$$

We denote the image group by $\bar{N}$ (note $Y^{*}=Y$ ). Then we consider the image of $I$ under the action of $G$ on $U(2)$ by linear fractional transformations. In this context to simplify the form subgroup $S$ we must apply a Cayley transform. If we set

$$
L=\frac{1}{\sqrt{2}}\left[\begin{array}{cc}
I & i I \\
I & -i I
\end{array}\right]
$$

then the transform is given by

$$
\sigma^{-1}(g)=L^{-1} g L
$$

Thus, in this incarnation, we take $S$ to be the set of elements of $P S U(2,2)$ as $G / \operatorname{Center}(\mathrm{G})$ in the form

$$
\left[\begin{array}{ll}
a I & b I \\
c I & d I
\end{array}\right]
$$

with $\operatorname{Im} a \bar{b}=0, a \bar{b}-b \bar{c}=1, \operatorname{Im} c \bar{d}=0$. This implies that the image of $S \cap \bar{N}$ is the set of matrices

$$
\left[\begin{array}{ll}
I & 0 \\
c I & I
\end{array}\right]
$$

with $c \in \mathbb{R}$. Let $z=\left(k_{1}, k_{2}, k_{3}, \omega\right)$ be, as above, an element in the light cone. Then considering $z$, as giving a linear functional on $\operatorname{Lie}(\bar{N})$, this functional restricted to $\left[\begin{array}{cc}0 & 0 \\ x I & 0\end{array}\right] \in \operatorname{Lie}(S \cap \bar{N})$ is given by the coefficient of $q$ in the quadratic polynomial

$$
\frac{1}{2} \operatorname{det}\left[\begin{array}{cc}
\omega+k_{3}+q x & k_{1}+i k_{2} \\
k_{1}-i k_{2} & \omega-k_{3}+q x
\end{array}\right]
$$

which is $\omega x$. We will record this as a lemma since we will need to apply it later in this section. 
Lemma 25 If $z=\left(k_{1}, k_{2}, k_{3}, \omega\right)$ is in the null light cone and if we consider $z$ as a linear functional on $\operatorname{Lie}(\bar{N})$ (as above), then its value on

$$
\left[\begin{array}{cc}
0 & 0 \\
x I & 0
\end{array}\right]
$$

is $\omega x$.

We now relate these plane wave solutions to the representations $\mathrm{Maxw}_{\alpha, b}^{ \pm}$ with $(\alpha, \beta) \in\{(2,0),\{0,2)\}$. Let $H_{\alpha, \beta}^{ \pm}$be the corresponding Hilbert space completions of these smooth Fréchet representations of moderate growth. Then the $\mathrm{Maxw}_{\alpha, b}^{ \pm}$are the spaces of $C^{\infty}$ vectors. Fix one of these representations and denote it by $(\pi, H)$. Let $H_{K}$ be the space of $K$-finite vectors and let $\langle v, w\rangle_{k}=\left\langle\left(I+C_{K}\right)^{k d} v, w\right\rangle$ for $v, w \in H_{K},\langle\ldots, \ldots\rangle$ the unitary structure and $d=\operatorname{dim} K$. Let $H^{k}$ be the Hilbert space completion of $H_{K}$ with respect to $\langle\ldots, \ldots\rangle_{k}$. Then $H^{0}=H$ and the maps $T_{k, l}: H^{l} \rightarrow H^{k}$ for $k>l \geq 0$ that are the identity on $H_{K}$ are nuclear (see [GV] for the definition). Thus we have $\cap_{k \geq 0} H^{k}=H^{\infty}$. We set $H^{-k}$ equal to the dual Hilbert space to $H^{k}$. Thus $\left(H^{\infty}\right)^{\prime}=\cup_{k \geq 0} H^{-k}$. We thus have a rigged Hilbert space in the sense of $[\mathrm{GV}]$, Chapter 1 . We now consider $(\pi, H)$ as a unitary representation of $\bar{N}$. We have (c.f. [W1], Theorem 14.10.3) a direct integral decomposition of $(\pi, H)$ as a representation of $\bar{N}$ given as follows:

$$
\int_{\operatorname{supp}(\pi) \subset \widehat{\bar{N}}} \mathbb{C}_{\chi} \otimes W_{\chi} d \tau(\chi)
$$

with $\operatorname{supp}(\pi)$ defined as in [W1, Volume 2, p. 337], $\tau$ a Borel measure on $\operatorname{supp}(\pi)$, and $\chi \rightarrow W_{\chi}$ a Borel measurable Hilbert vector bundle over $\operatorname{supp}(\pi)$. In this case $\operatorname{supp}(p)$ is just the support of $\tau$ as a distribution. The theorem of Gelfand-Kostyuchenko (c.f. [GV], p. 117 Theorem 1' and the remarks at the end of Subsection 1.4.4) implies that there exists, $k>0$ and a family of nuclear operators $T_{\chi}: H^{k} \rightarrow W_{\chi}$ such that if $u \in H^{k}$, then $u(\chi)=T_{\chi}(u)$ for $\tau$-almost all $\chi$. This implies that for all $\chi$ and all $u \in H^{k}$,

$$
T_{\chi}(\pi(\bar{n}) u)=\chi(\bar{n}) T_{\chi}(u) .
$$

If $T_{\chi}$ is always 0 then $H=0$. We see that there is a subset $A$ of $\operatorname{supp}(\pi)$ of full measure with $T_{\chi} \neq 0$ for $\chi \in A$. By definition of the $H^{k}$ we see that if $\chi \in A$, then there exists $\lambda \in W_{\chi}^{\prime}$ such that $\lambda \circ T_{\chi} \neq 0$. We have for this choice

$$
\lambda \circ T_{\chi}(\pi(\bar{n}) u)=\chi(\bar{n}) \lambda \circ T_{\chi}(u)
$$


for all $\bar{n} \in \bar{N}$ and all $u \in H^{\infty}$. If $\chi \in \widehat{\bar{N}}$, then set

$$
W h_{\chi}(\pi)=\left\{\mu \in\left(H^{\infty}\right)^{\prime} \mid \mu \circ \pi(\bar{n})=\chi(\bar{n}) \mu\right\} .
$$

We have with this notation

Theorem 26 Let $H^{\infty}$ be one of $\mathrm{Maxw}_{2,0}^{ \pm}$or $\mathrm{Maxw}_{2,0}^{ \pm}$. Then

1. $\operatorname{dim} W h_{\chi}(\pi) \leq 3$.

2. Writing

$$
\chi_{Z}\left(\left[\begin{array}{cc}
I & 0 \\
Y & I
\end{array}\right]\right)=e^{i(Z, Y)}
$$

with $Z^{*}=Z$ (this describes all possible $\left.\chi\right)$. Then $W h_{\chi_{Z}}(\pi)=0$ if $\operatorname{det}(Z) \neq 0$ (i.e., the support of $\pi$ is contained in the null light cone). Furthermore, the support of $\pi$ is the closure of a single orbit of the action of $G L(2, \mathbb{C})$ on $\left\{Z \in M_{2}(\mathbb{C}) \mid Z^{*}=Z\right\}$ given by $g \cdot Z=g Z g^{*}$.

3. Both $\pi_{2,0}^{+}, \pi_{0,2}^{+}$have support equal to the set of all $\chi_{Z}$ with $\operatorname{det} Z=0$ and $\operatorname{tr} Z \leq 0$ (negative light cone) and both $\pi_{2,0}^{-}, \pi_{2,0}^{-}$have support equal to the set of all $\chi_{Z}$ with $\operatorname{det} Z=0$ and $\operatorname{tr} Z \geq 0$ (positive light cone).

Note: The proof of this result will use earlier work of the authors $[\mathrm{K}],[\mathrm{W} 2],[\mathrm{W} 3]$.

Proof. We will do all the details for $\mathrm{Maxw}_{2,0}^{+}$. The case $\mathrm{Maxw}_{0,2}^{+}$is essentially the same. The cases $\mathrm{Maxw}_{2,0}^{-}$and $\mathrm{Maxw}_{0,2}^{-}$are done with an interchange of $\mathfrak{p}^{ \pm}$with $\mathfrak{p}^{\mp}$. Set $\mathfrak{q}=\mathfrak{k} \oplus \mathfrak{p}^{+}$where $\mathfrak{k}=\operatorname{Lie}(K)_{\mathbb{C}}$. We consider $F^{2,0,2}$ to be the $\mathfrak{q}$-module with $\mathfrak{k}$ acting through the differential of the $K$ action and $\mathfrak{p}^{+}$ acting by 0 . Set

$$
N\left(F^{2,0,2}\right)=U(\mathfrak{g}) \otimes_{U(\mathfrak{q})} F^{2,0,2} .
$$

Then Lemma 18 implies that we have a surjective $(\mathfrak{g}, K)$-module homomorphism

$$
\left.p: N\left(F^{2,0,2}\right) \rightarrow \mathrm{Maxw}_{2,0}^{+}\right)_{K} .
$$

Thus $p^{*} W h_{\chi}\left(\pi_{2,0}^{+}\right)$is contained in

$$
\mathcal{W}_{\chi}=\left\{\lambda \in \operatorname{Hom}_{\mathbb{C}}\left(N\left(F^{2,0,2}\right), \mathbb{C}\right) \mid \lambda(Y v)=d \chi(Y) \lambda(v), Y \in \operatorname{Lie}(\bar{N})\right\} .
$$

Since $p^{*}: W h_{\chi}\left(\pi_{2,0}^{+}\right) \rightarrow \mathcal{W}_{\chi}$ is injective $\left(\left(\operatorname{Maxw}_{2,0}^{+}\right)_{K}\right.$ is dense in $\left.\operatorname{Maxw}_{2,0}^{+}\right)$ the dimension estimate will follow if we prove that $\operatorname{dim} \mathcal{W}_{\chi}=3$. For this we use the observation in [W3] that if

$$
L=\left\{\left[\begin{array}{cc}
g & 0 \\
0 & \left(g^{-1}\right)^{*}
\end{array}\right] \mid g \in G L(2, \mathbb{C})\right\},
$$


then $\operatorname{Lie}(L \bar{N})_{\mathbb{C}}$ and $\mathfrak{q}$ are opposite parabolic subalgebras (i.e., $\operatorname{Lie}(L \bar{N})_{\mathbb{C}} \cap \mathfrak{q}$ is a Levi factor of both parabolic subalgebras). This implies that $N\left(F^{2,0,2}\right)$ is a free $\operatorname{Lie}(\bar{N})_{\mathbb{C}}$ module on $\operatorname{dim} F^{2,0,2}=3$ generators. This clearly implies that $\operatorname{dim} \mathcal{W}_{\chi}=3$.

We now note if $m=\left[\begin{array}{cc}g & 0 \\ 0 & \left(g^{-1}\right)^{*}\end{array}\right]$ then $m\left[\begin{array}{cc}I & 0 \\ Y & I\end{array}\right] m^{-1}=\left[\begin{array}{cc}I & 0 \\ g \cdot Y & I\end{array}\right]$. Set $\bar{n}=\bar{n}(Y)=\left[\begin{array}{cc}I & 0 \\ Y & I\end{array}\right]$. If $\lambda \in W h_{\chi_{Z}}\left(\pi_{2,0}^{+}\right)$, then

$$
\begin{gathered}
\lambda \circ \pi_{2,0}^{+}(m)\left(\pi_{2,0}^{+}(\bar{n}) u\right)=\lambda\left(\pi_{2,0}^{+}(m) \pi_{2,0}^{+}(\bar{n}) u\right) \\
=\lambda\left(\pi_{2,0}^{+}\left(m \bar{n} m^{-1}\right) \pi_{2,0}^{+}(m) u\right)=\chi_{Z}\left(m \bar{n} m^{-1}\right) \lambda \circ \pi_{2,0}^{+}(m)(u) \\
=\chi_{g^{-1} \cdot Z}(\bar{n}) \lambda \circ \pi_{2,0}^{+}(m)(u) .
\end{gathered}
$$

Thus $\lambda \circ \pi_{2,0}^{+}(m) \in W h_{\chi_{g^{-1} Z}}\left(\pi_{2,0}^{+}\right)$. This implies that $\operatorname{supp}\left(\pi_{2,0}^{+}\right)$is a union of orbits.

We now observe using results in [W3] that we can show that if $W h_{\chi_{Z}}\left(\pi_{2,0}^{+}\right) \neq$ 0 with $\operatorname{det} Z \neq 0$, then the $G K$-dimension of $\left(\mathrm{Maxw}_{2,0}^{+}\right)_{K}$ is at least $\operatorname{dim} \bar{N}=4$. Indeed, this condition implies that the dual module to $\left.\mathrm{Maxw}_{2,0}^{+}\right)_{K}$ contains an irreducible submodule of $G K$-dimension 4. However as a $\mathfrak{p}^{-}$-module $\left(\mathrm{Maxw}_{2,0}^{+}\right)_{K}$ is graded with the $k$-th level of the grad being isomorphic with the $K$-module $F^{k+2, k, k+2}$ whose dimension is $(k+3)(k+1)$. Thus the $G K$ dimension of $\left(\mathrm{Maxw}_{2,0}^{+}\right)_{K}$ is 3 . Thus we must have $\operatorname{det} Z=0$. To complete the proof, it is enough to prove 3 . Since there are three orbits of $L$ in set of all $Z$ with $\operatorname{det} Z=0$ :

$$
\mathcal{O}^{+}=\{Z \mid \operatorname{det} Z=0, \operatorname{tr} Z>0\}, \mathcal{O}^{+}=\{Z \mid \operatorname{det} Z=0, \operatorname{tr} Z<0\},\{0\} .
$$

Thus we must prove that if $W h_{\chi_{Z}}\left(\pi_{2,0}^{+}\right) \neq 0$ and $Z \neq 0$ then $\operatorname{tr} Z<0$. This follows from Theorem 17 (p. 306) in [W2] (in this reference the roles of $\chi$ and $\chi^{-1}$ are reversed). We can also prove the result directly using Lemma 25. Let $\lambda \neq 0$ be an element of $W h_{\chi_{Z}}\left(\pi_{2,0}^{+}\right)$with $Z \neq 0$. Then set $\eta=d \chi_{Z}$. If

$$
Z=\left[\begin{array}{cc}
\omega+k_{3} & k_{1}+i k_{2} \\
k_{1}-i k_{2} & \omega-k_{3} x
\end{array}\right],
$$

then $\operatorname{tr} Z=2 \omega$. In Lemma 25 we saw that

$$
\eta\left[\begin{array}{cc}
0 & 0 \\
x I & 0
\end{array}\right]=\omega x .
$$


This if $S$ is as in the previous section and $\nu=\chi_{\mid N \cap S}$ then in the notation of [W2] Theorem 2, $r_{\nu}=\omega$. Thus that theorem (also see $[\mathrm{K}]$ ) implies that (taking into account the reversal of signs mentioned above) $r_{\nu}<0$. This completes the proof.

Remark 27 The above results imply that in the steady state solutions to Maxwell's equations the plane wave solutions should be looked upon as 2-currents. That is, using $\gamma$, they are distributions on Maxw.

\section{Conclusion}

In this paper we have shown (Theorem 19) that the solutions to Maxwell's equations that extend to the conformal compactification of Minkowski space break up into four irreducible unitary representations: 2 positive energy and 2 negative energy (in the sense of lowest weight or highest weight respectively) as a representation of the conformal group of the wave equation. The support of each of the representations is either the forward or backward null light cone. The ones with positive energy (Theorem 26) yield only positive frequencies and thus according to Planck's law have positive energy in the sense of field theory. We observe that solutions that extend to the total compactification (i.e., steady state) can have their frequency spectrum constrained to a narrow band yielding background radiation that indicates a temperature of 2.7 degrees Kelvin (or any other temperature for that matter). This says that although the actual steady state models to the universe that fit the astronomical observations are complicated this work indicates that there can be a background radiation that fits the measurements that is not the outgrowth of an initial very high temperature source. We would also like to point out that the big bang models for the universe have had difficulty fitting the observations also, leading to theories involving inflation and the return of the cosmological constant. An interesting alternative which is not unrelated to this paper can be found in $[\mathrm{P}]$ where the conformal structure is emphasized and time does almost cycle. There is also the chronometric theory of $[\mathrm{S}]$.

We have also shown that these representations fit in larger contexts. How-

ever, although many of the results in this paper generalize to $S O(n, 2)$, the beautiful geometric structures that appear in the case $n=4$ do not. 


\section{References}

[ BW] A. Borel and N. Wallach, Continuous cohomology, discrete subgroups, and representations of reductive groups, Second edition. Mathematical Surveys and Monographs, 67. American Mathematical Society, Providence, RI, 2000 .

[ EW] T. Enright and N. Wallach, Embeddings of unitary highest weight representations and generalized Dirac operators, Math. Ann. 307 (1997), 627-646.

[ GV] I. M. Gelfand and N. Y.Vilenkin, Generalized Functions Vol. 4: Applications of Harmonic Analysis, Academic Press, 1964.

[HBN] F. Hoyle, G. Burbidge, J.V. Narlikar, A quasi-steady state cosmological model with creation of matter, The Astrophysical Journal 410(1993), 437-457.

[ GW] B. Gross and N. Wallach, On quaternionic discrete series representations, and their continuations, J. Reine Angew. Math., 481(1996), 73-123.

[ HSS] M. Hunziker, M. Sepanski and R. Stanke, Conformal symmetries of the wave operator, to appear.

[ K] B. Kostant, On Laguerre polynomials, Bessel functions, Hankel transform and a series in the unitary dual of the simply-connected covering group of $S L(2, \mathbb{R})$, Represent. Theory 4 (2000), 181-224.

[ P] Roger Penrose, Cycles of Time, Alfred Knopf, New York, 2011.

[S] Irving Segal, Radiation in the Einstein universe and the cosmic background, Physical Review D 28(1983), 2393-2402.

[ VZ] D. Vogan and G. Zuckerman, Unitary representations and continuous cohomology, Comp/ Math. 53 (1984), 51-90.

[ W1] Nolan R. Wallach, Real reductive groups I,II, Academic Press, New York, 1988,1992.

[W2] N. Wallach, Generalized Whittaker vectors for holomorphic and quaternionic representations, Comment. Math. Helv. 78 (2003), 266-307.

[ W3] N.R. Wallach, Lie algebra cohomology and holomorphic continuation of generalized Jacquet integrals, Advance Studies in Pure Math 14 (1988),123151. 